High Brightness Plasma-Based Soft X-ray Lasers and Applications

J. Dunn

December 30, 2009

Frontiers in Optics 2009/Laser Science XXV San Jose, CA, United States October 13, 2009 through October 13, 2009 
This document was prepared as an account of work sponsored by an agency of the United States government. Neither the United States government nor Lawrence Livermore National Security, LLC, nor any of their employees makes any warranty, expressed or implied, or assumes any legal liability or responsibility for the accuracy, completeness, or usefulness of any information, apparatus, product, or process disclosed, or represents that its use would not infringe privately owned rights. Reference herein to any specific commercial product, process, or service by trade name, trademark, manufacturer, or otherwise does not necessarily constitute or imply its endorsement, recommendation, or favoring by the United States government or Lawrence Livermore National Security, LLC. The views and opinions of authors expressed herein do not necessarily state or reflect those of the United States government or Lawrence Livermore National Security, LLC, and shall not be used for advertising or product endorsement purposes. 


\title{
High Brightness Plasma-Based Soft X-ray Lasers and Applications
}

\author{
James Dunn a) \\ Physics Division, Physical Life Sciences Directorate, \\ Lawrence Livermore National Laboratory, Livermore, CA 94551, \\ USA

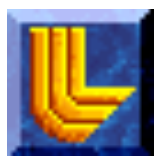 \\ LLNL-CONF-422049
}

Invited Talk presented at the $93^{\text {rd }}$ Annual Meeting of the Optical Society of America

Frontiers in Optics 2009/Laser Science XXV, 13 October 2009, San Jose, CA

a)dunn6@IInl.gov

This work performed under the auspices of the U.S. Department of Energy by Lawrence Livermore National Laboratory under Contract DE-AC52-07NA27344, through the Institute of Laser Science and Applications and in part sponsored by the National Nuclear Security Administration under the Stewardship Science Academic Alliances program through U.S. Department of Energy Research Grant \#DEFG52-06NA26152. 
Acknowledgments to contributions, information and discussions with numerous colleagues:

S. Moon, R. Keenan, R. Smith, A. Nelson, J. Nilsen, R. Shepherd

XRL Characterization, applications, experiments, simulations

J. Rocca, M. Marconi, J. Filevich, M. Purvis, J. Grava X-ray Laser Interferometry

(Colorado State University)

V. Shlyaptsev, H. Baldis

Simulations and new pumping geometry, Thomson Scattering

B. Rus, T. Mocek, ...

X-ray Thomson Scattering

(Inst. Of Physics, Czech Republic)

Special thanks to colleagues for presenting work from their research groups:

S. Sebban, P. Zeitoun(LOA, France) Higher order Harmonic (HOH) seeding

J. Rocca, C. Menoni, M. Marconi (CSU, USA) HOH seeding XRLs and applications 


\section{Overview:}

- History, introduction and description of plasma-based soft x-ray lasers

- Characteristics

- Recent developments in source generation: Smaller pump energy, higher repetition rate, improved beam qualities

- Examples of applications: Interferometry, microscopy, ablation studies, imaging, probing matter

- Summary

Notes:

- Soft x-ray lasers are ultra-bright $10^{24}-10^{27}$ ph. mm-2 mrad $^{-2} \mathrm{~s}^{-1}[0.1 \% \mathrm{BW}]^{-1}$

- Latest generation are very compact (table top), inexpensive compared to synchrotrons, and are compatible with small group and university research

- Complement 3rd and 4th generation synchrotron sources for wavelength range, applications and regimes of interest 


\section{Brief History: Laboratory X-ray Laser is 25 years old}

- Plasma-based x-ray lasers description

- Novette and Nova Laser X-ray Laser Program at LLNL 
Soft x-ray laser requires creating plasma column with closed shell $\mathrm{Ni}$-like ion and collisionally pumping 4d upper level

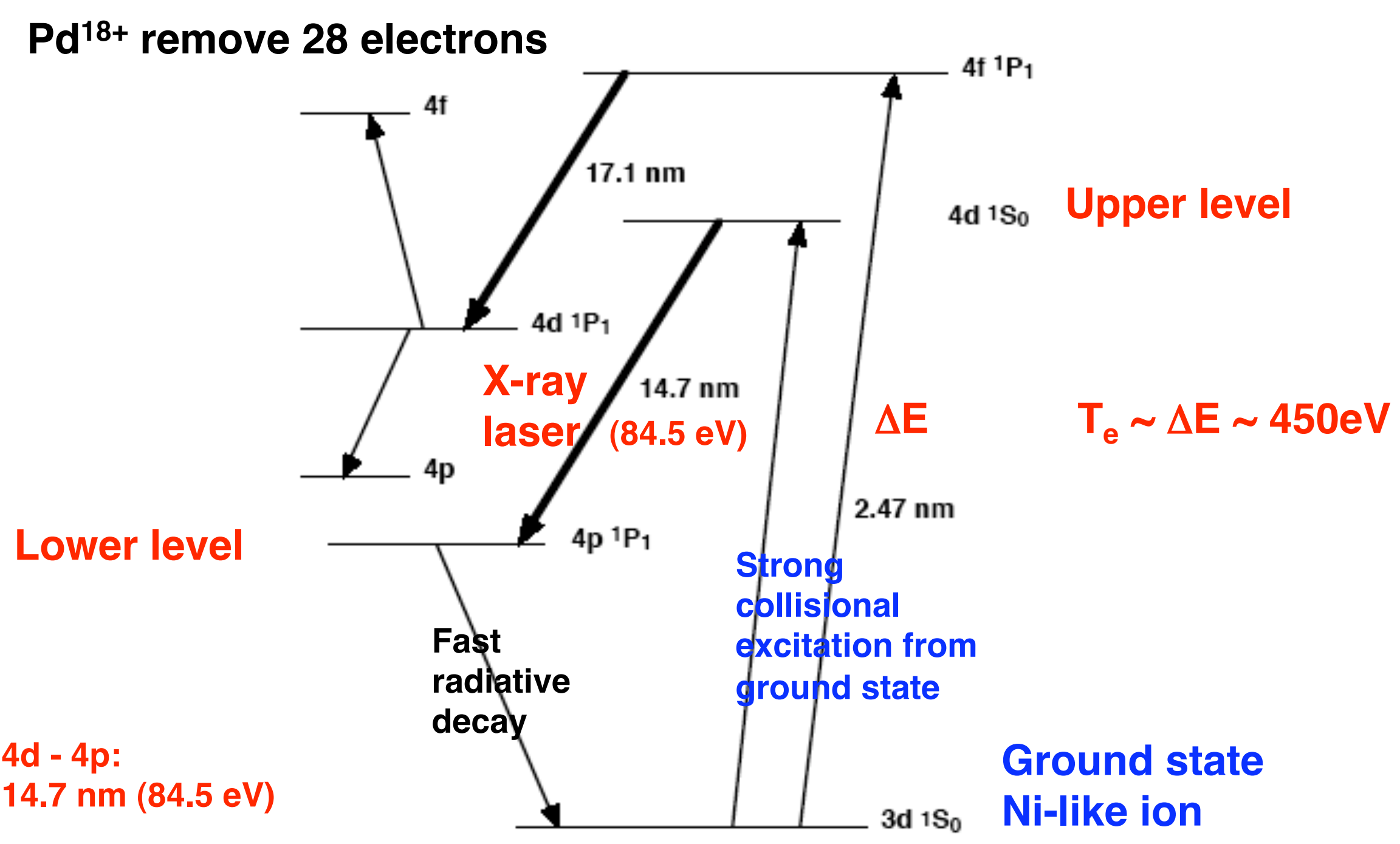

Ni-like ion (gain medium) has a closed shell configuration remains stable over a range of plasma conditions. 
X-ray laser photon energy is fixed (discrete) - dependent on energy level structure of excited states of highly-charged ions

Wavelength of $4 d \rightarrow 4 p$ lasing transition scales as $\lambda \sim 1 / Z^{3}$ for the Ni-like schemes

Power to pump XRL scales as $P \sim 1 / \lambda^{4-6}$

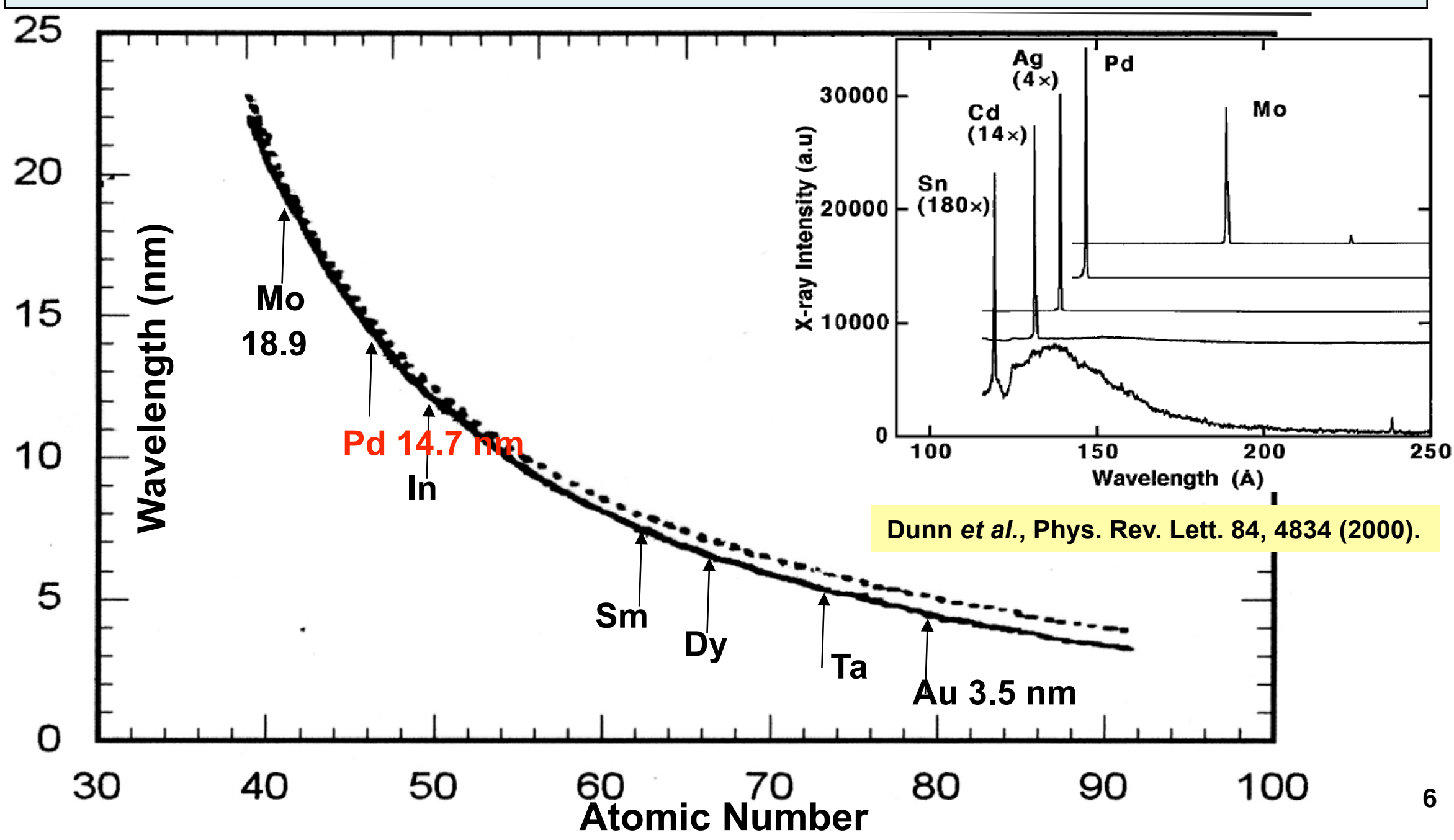


Early effort 1984 - 1996 on x-ray lasers was performed on the Nova laser: Collisional excitation scheme was developed

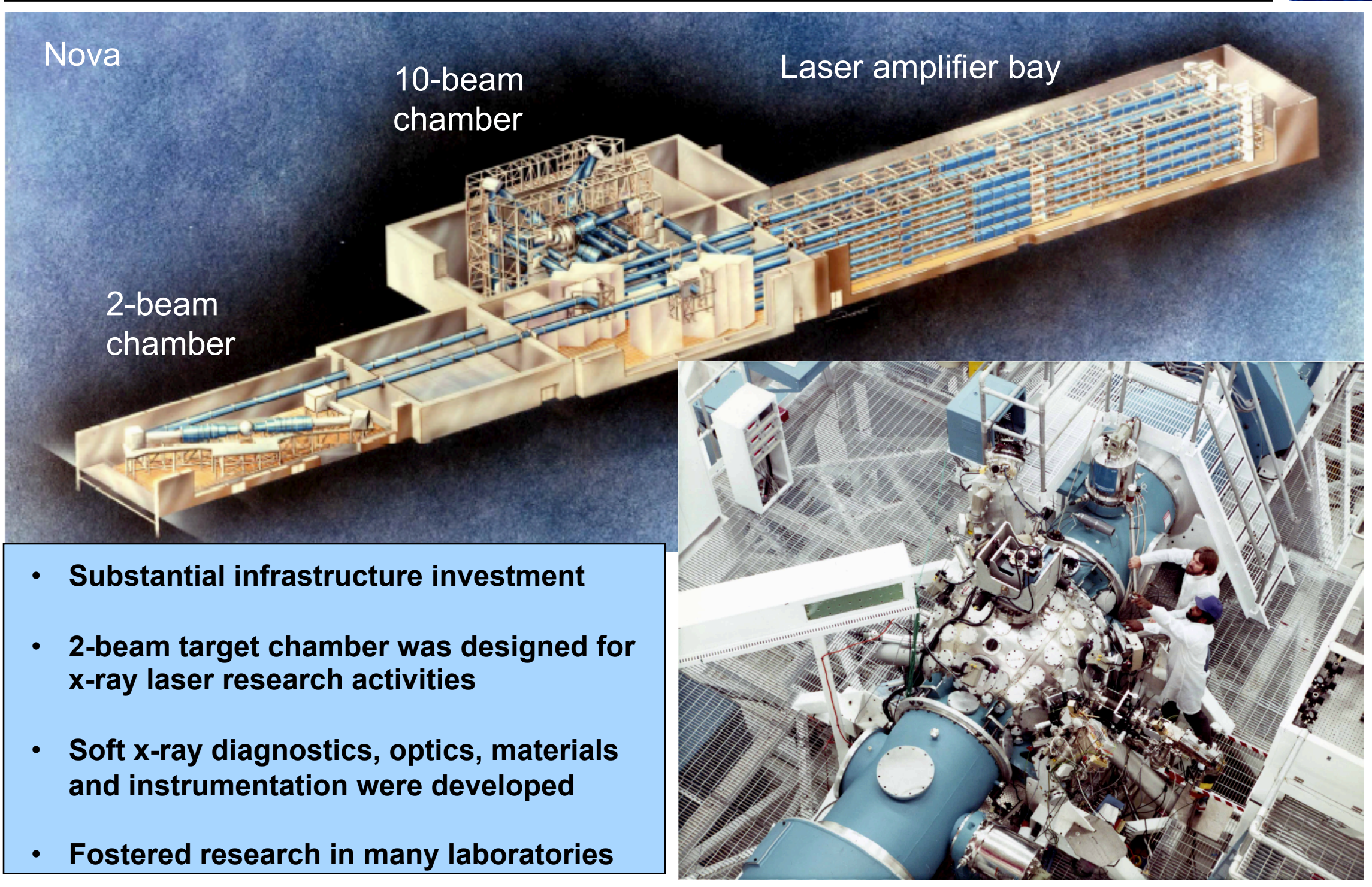




\section{Exploding foil target and x-ray laser was designed after substantial modeling and experimental characterization effort}

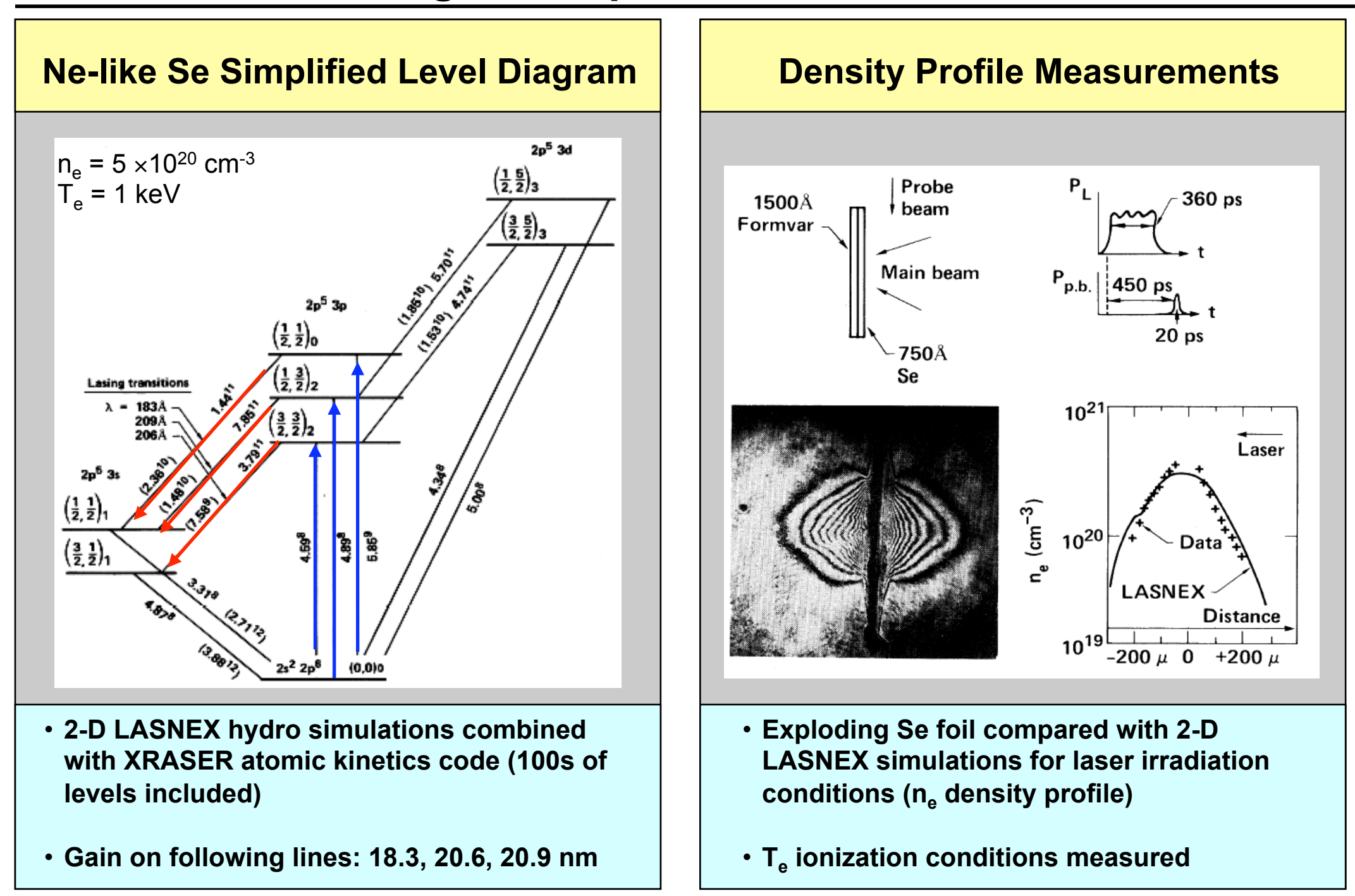


$\mathrm{Ne}$-like Se laser at $\sim 20 \mathrm{~nm}$ was first demonstration of collisional excitation x-ray laser in 1984 on Novette

- Exploding foil Se target

- $1 \mathrm{~kJ}, 450$ ps $2 \omega$ each side

- Line focus $200 \mu \mathrm{m} \times 1.1 \mathrm{~cm}, 2.2 \mathrm{~cm}$

- Double and single-sided irradiation
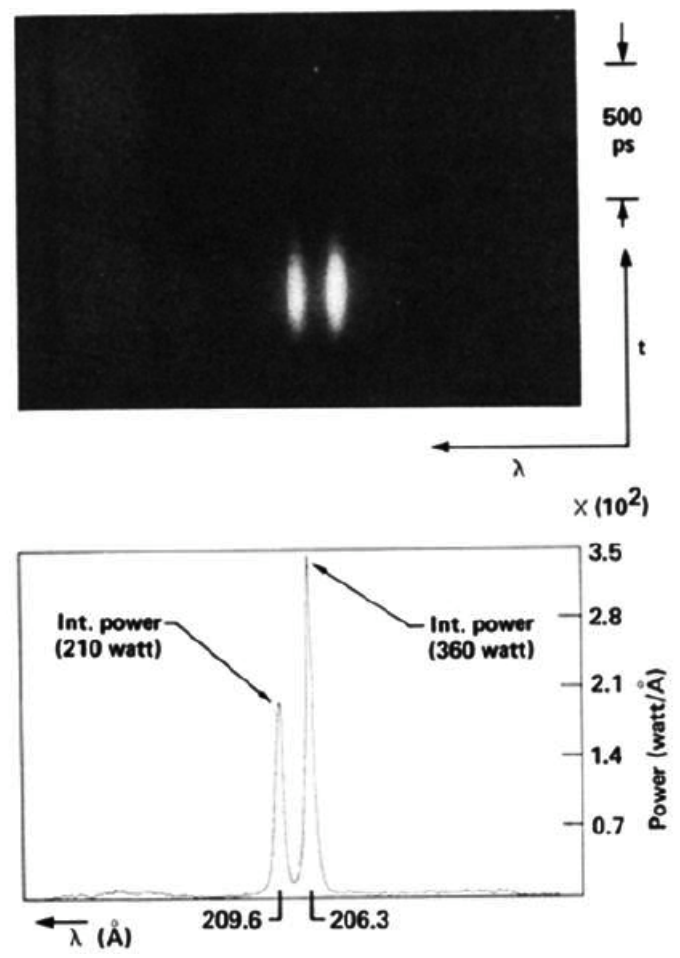

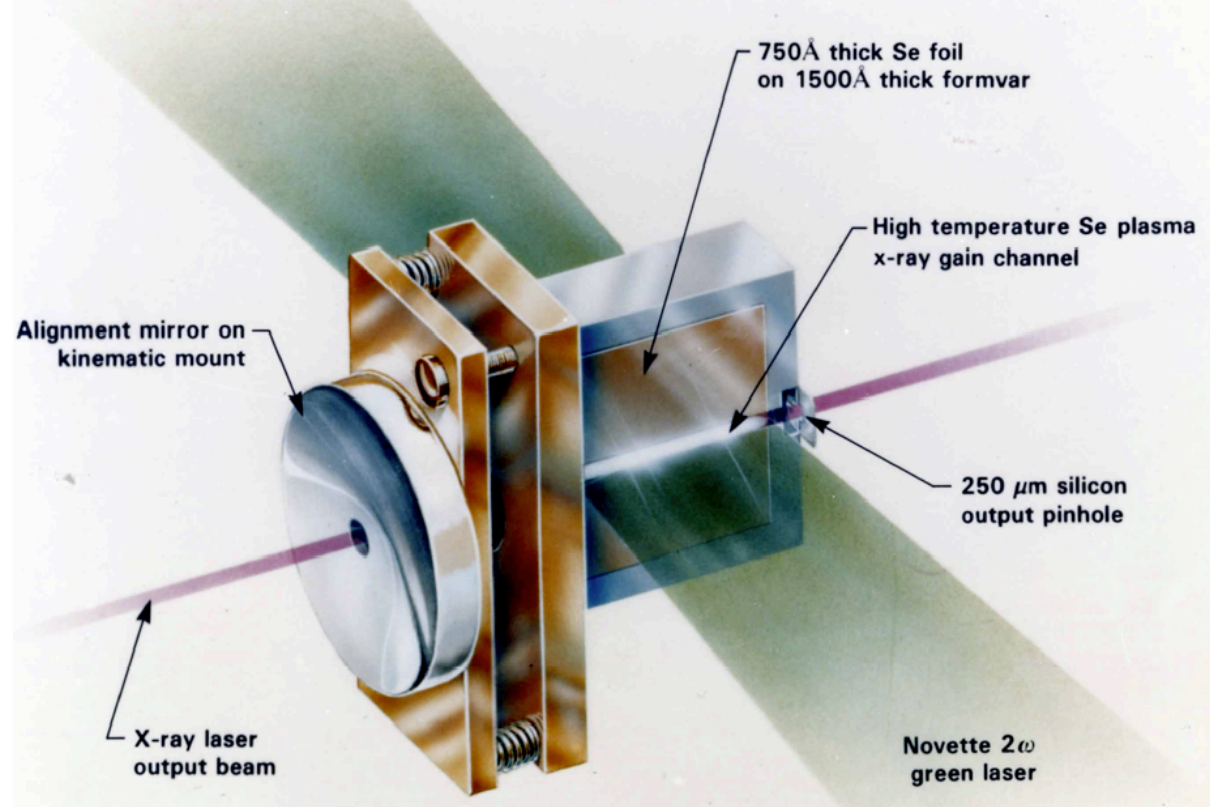

- Lasing observed on Ne-like $\mathrm{Y}$ transitions

- Lasing observed on Ne-like 3p - 3s J = 2 - 1 lines at 20.63 and $20.96 \mathrm{~nm}$

- $\mathrm{g} \sim 5 \mathrm{~cm}^{-1}, \mathrm{gL}=6.5$

- No lasing observed on $18.3 \mathrm{~nm} \mathrm{~J}=0-1$ line 


\section{Recent developments in X-ray Laser Sources}

- Tabletop Capillary Discharge

- Tabletop Laser-pumped Transient scheme

- X-ray Laser characteristics 


\begin{tabular}{|ll|}
\hline \multicolumn{2}{|c|}{ Capillary discharge $46.9 \mathrm{~nm}$ laser } \\
- High average power: & $1-3 \mathrm{~mW}$ \\
- High pulse energy: & $0.1 \mathrm{~mJ}-0.8 \mathrm{~mJ} @ 4 \mathrm{~Hz}$ \\
- Narrow spectral bandwidth: & $\Delta \lambda / \lambda=10^{-4}$ \\
- Beam directionality: & $\theta=\sim 5 \mathrm{mrad}$ \\
\hline
\end{tabular}

Highest average power compact coherent SXR light source available

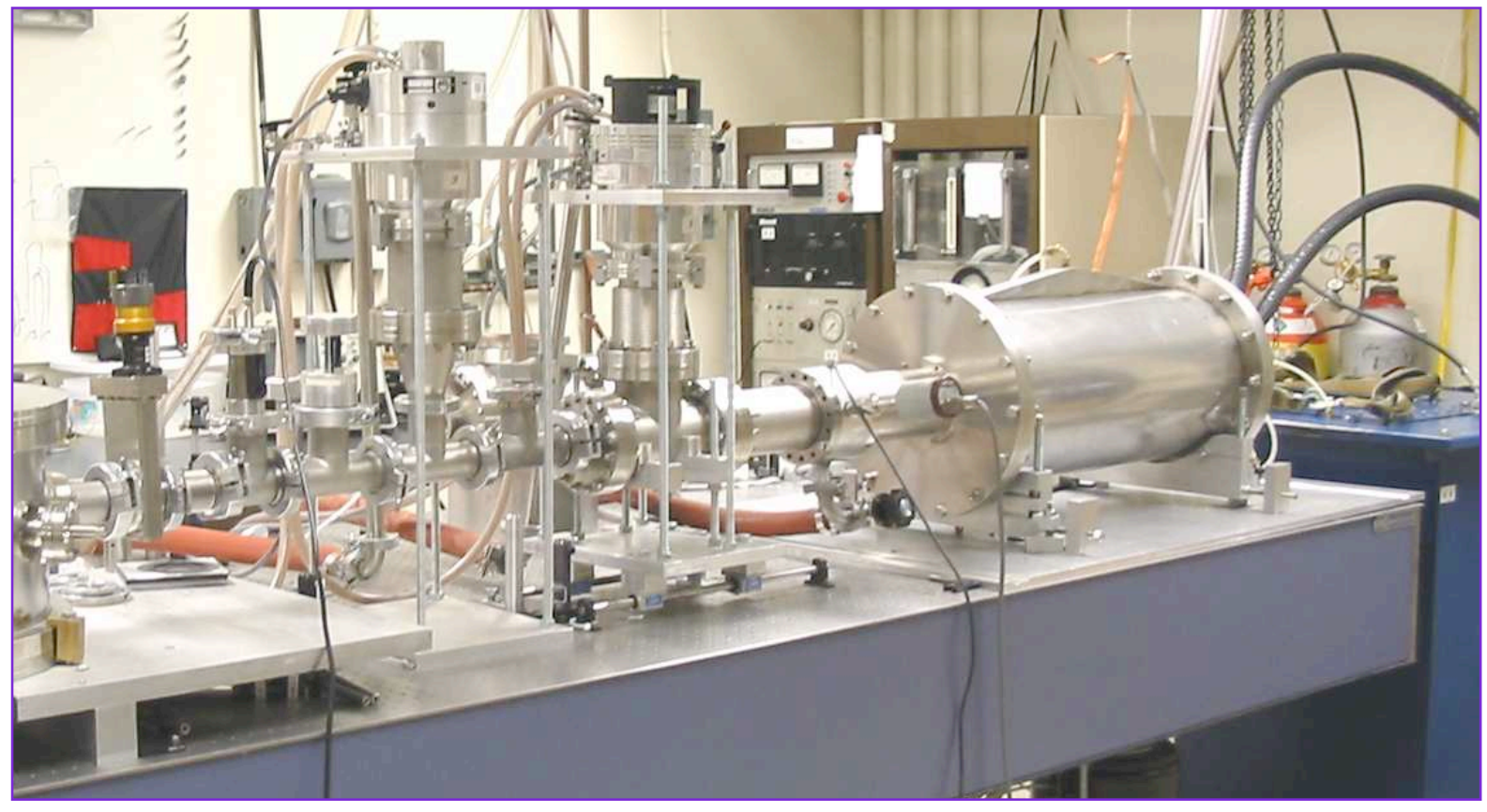

B. Benware et al., PRL $\underline{81}, 5804$ (1998): C.D. Macchietto et al., Opt. Lett. $\underline{24}, 1115$ (1999).

J. Rocca (CSU) 


\section{Major breakthrough: Transient scheme 1 ps, 5 - 10 TW laser pulse to optimize excitation - Tabletop X-ray Laser}

\section{Two Stage Process}

Long laser pulse: 1 - $2 \mathrm{~J}$

- plasma formation

- ionization

- delay for relaxation of density gradients

Short laser pulse: 2 - $5 \mathrm{~J}$

- excitation

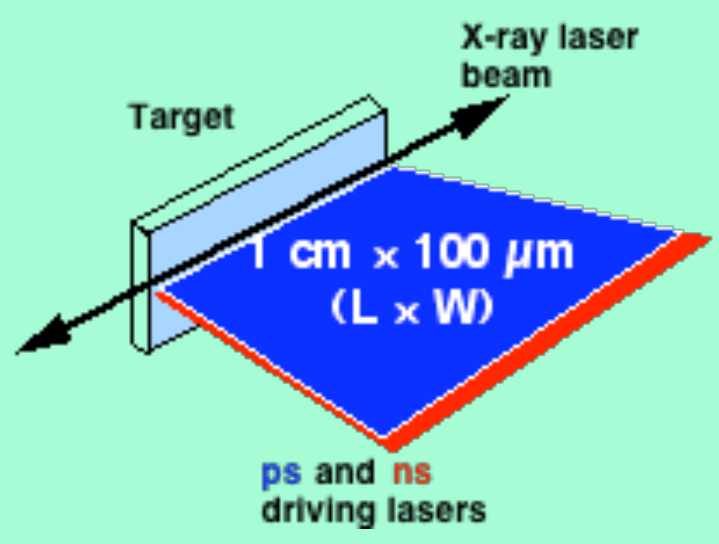

P.V. Nickles et al, PRL 78, 2748 (1997)

Yu. V. Afanasiev and V.N. Shlyaptsev, Sov. J. Quant. Electron. 19, 1606 (1989).

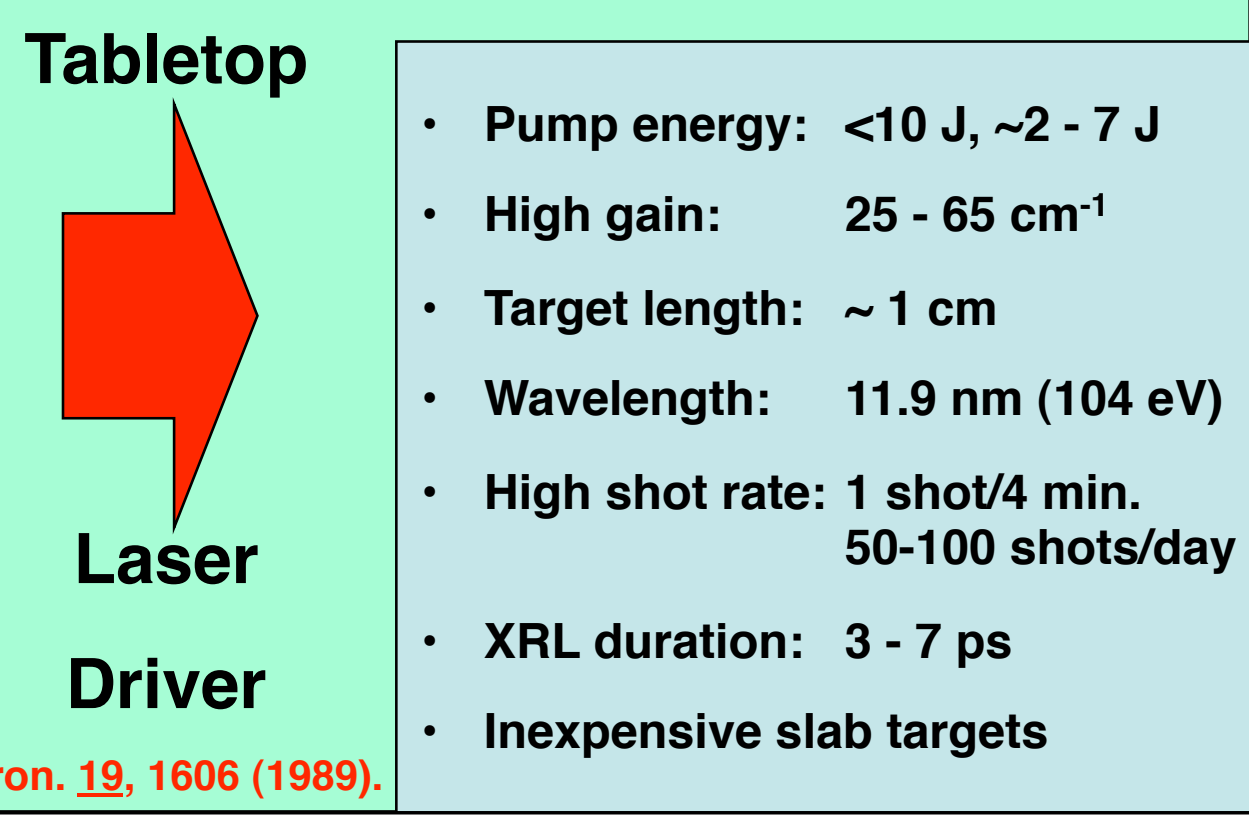

$10-1000 x$ reduction in laser energy for transient scheme (vs Nova) 


\section{LLNL COMET tabletop, laser-driven facility produced pulsed ps duration $x$-ray laser at 1 shot $/ 4$ minutes}
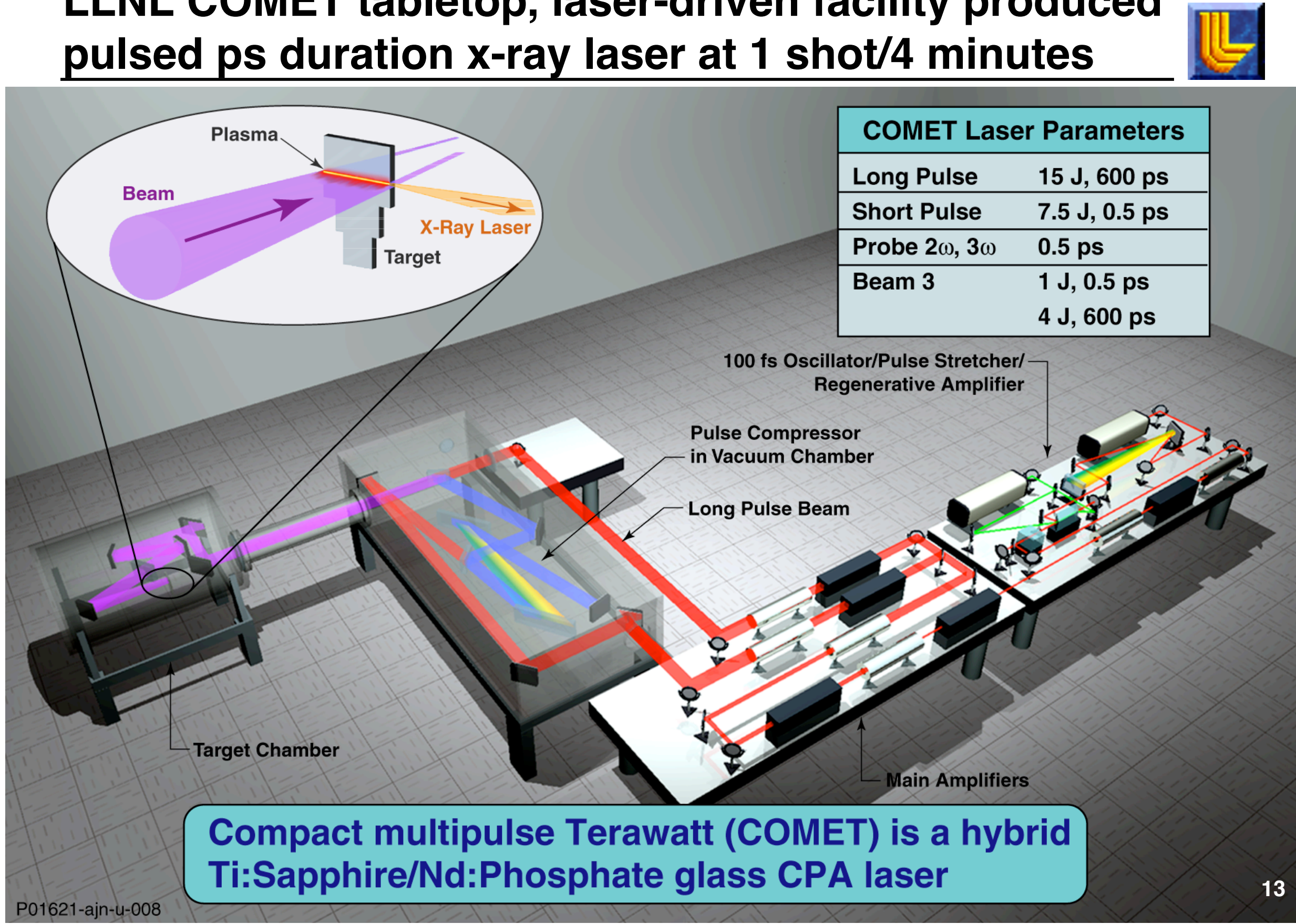


\section{Transient scheme produces pulsed ps duration x-ray laser - determined by gain lifetime in plasma}
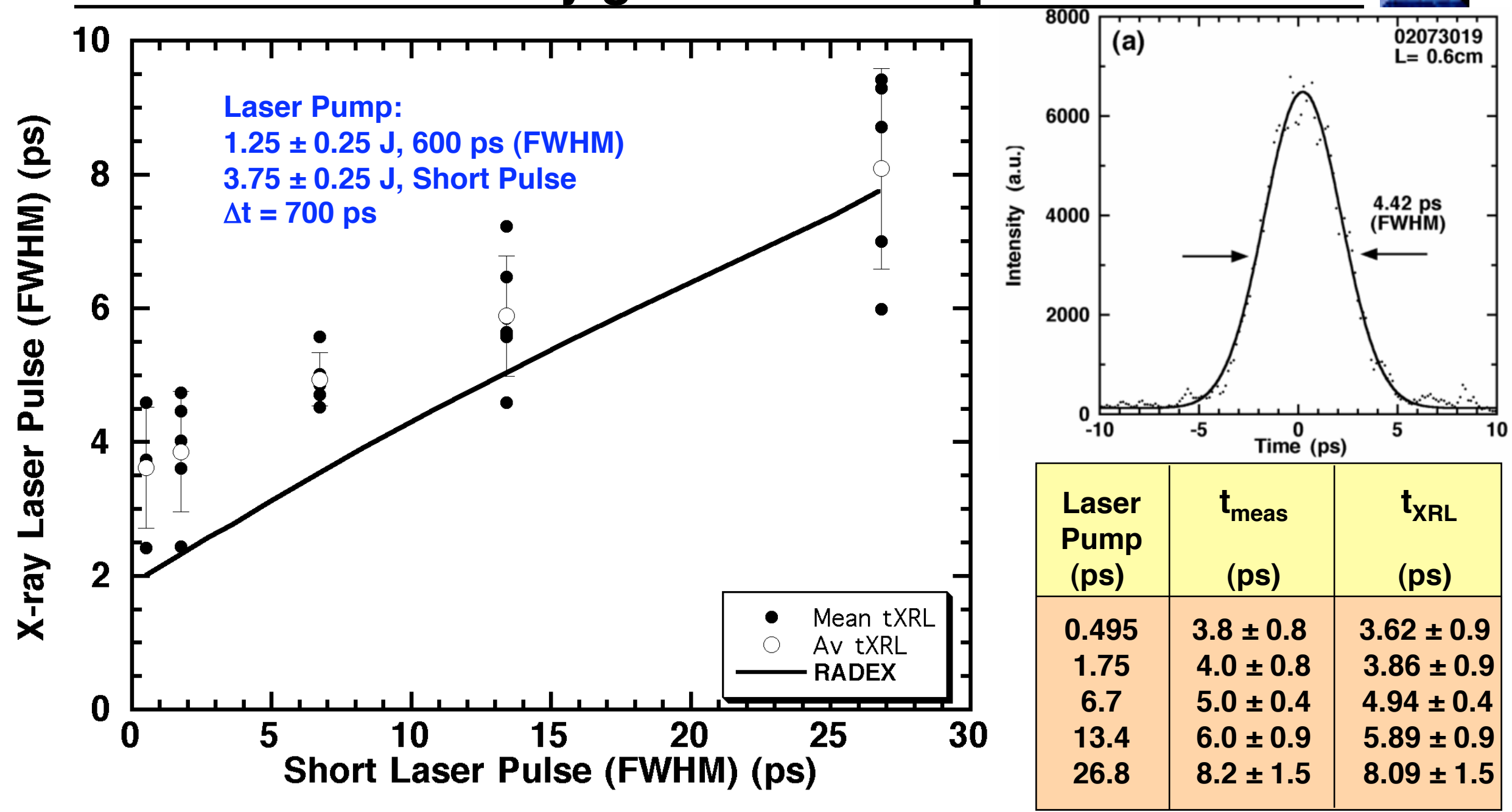

Dunn et al., SPIE Soft X-ray Lasers and Applications 2003.

- $t_{X R L}$ varies by $2.2 \times$ for $50 \times$ variation in short pulse

- Shortest observed $t_{X R L} 2.4 \pm 0.1$ ps (FWHM) for $<1.75$ ps short pulse 
Michelson Interferometer measured fringe visibility vs path difference for two pumping pulses 6 ps and 13 ps yields coherence length - spectral line width from FT

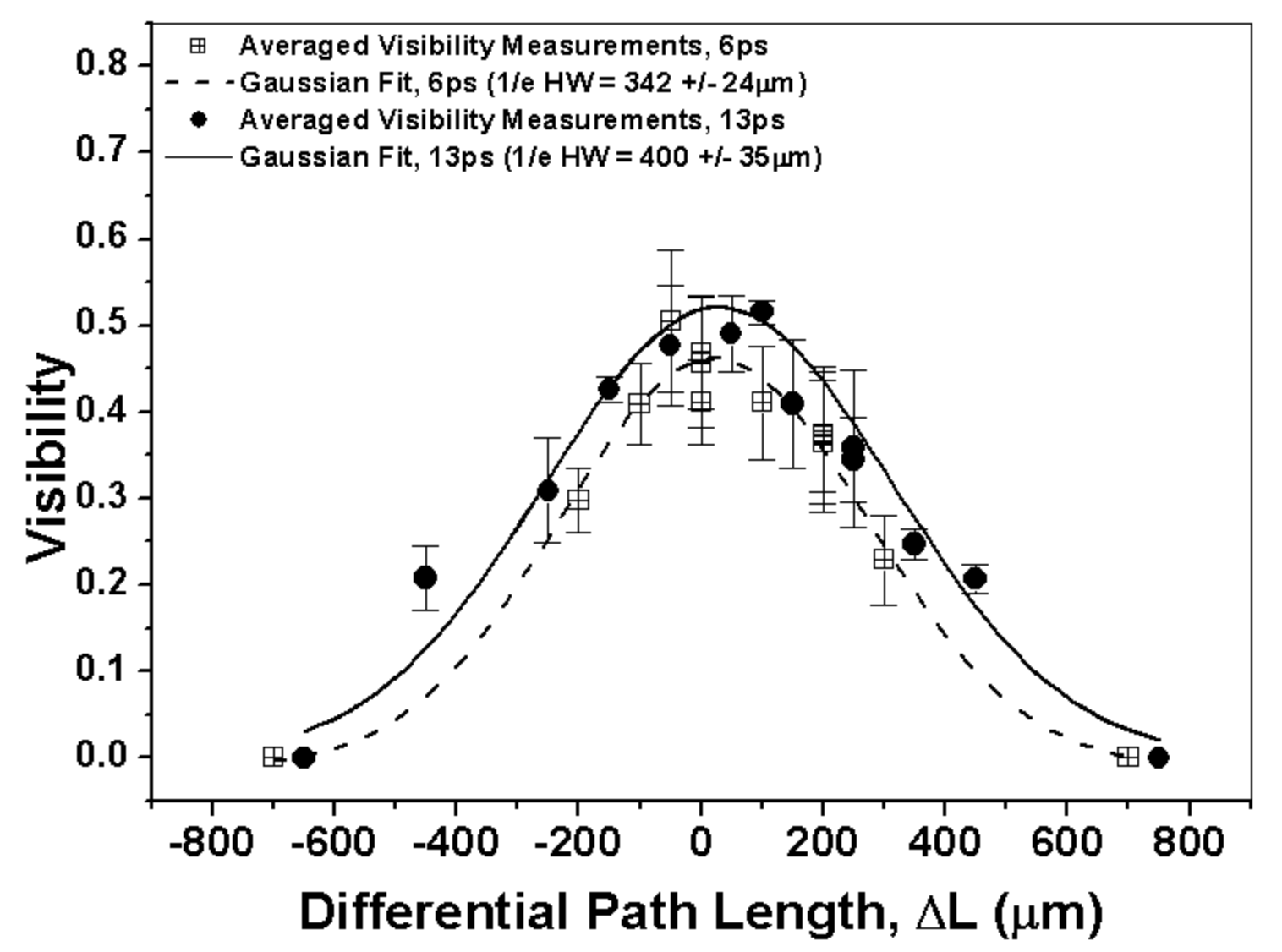

\begin{tabular}{l}
\multicolumn{1}{c|}{$\begin{array}{c}\text { Fringe visibility vs } \\
\text { path difference }\end{array}$} \\
Fringe visibility measured in same \\
three positions of interferogram from \\
shot-to-shot to improve statistics \\
$50 \%$ visibility results from unequal \\
throughput in two arms \\
Gaussian fit to data \\
$6 p s$ data: $342 \pm 24 \mu \mathrm{m} 1 / \mathrm{e}$ half-width \\
$13 p s$ data: $400 \pm 35 \mu \mathrm{m} 1 / \mathrm{e}$ half-width \\
Equivalent Gaussian spectral FWHM of \\
0.34 pm and 0.29 pm for 6 ps and $13 \mathrm{ps}$ \\
$(\lambda \Delta \lambda=43000$ and 50600 for $14.7 \mathrm{~nm})$
\end{tabular}

In collaboration with $\mathrm{P}_{\text {. Zeitoun }}$

Measured time response of 4.5 ps saturated $x$-ray laser indicates $4 x$ transform limit - $2 x$ for unsaturated output 


\section{Summary of Present Soft X-ray (EUV) Laser Characteristics:}

- Collisional excitation soft $x$-ray lasers:

$\lambda \sim 3.5 \mathrm{~nm}-60 \mathrm{~nm}(\mathrm{E} \sim 400 \mathrm{eV}-20 \mathrm{eV})$

- Characteristics:

- Gain saturation

Fs- Ps-Driven X-ray Lasers

PALS

$10 \mu \mathrm{J}$ output, $10^{12}$ photons/pulse

$10^{15} \mathrm{ph}$.

- High gain $30-80 \mathrm{~cm}^{-1}$ (small signal gain)

- Repetition rate

1 - $10 \mathrm{~Hz}$ (100 Hz DPSSL proposed by MBI, Berlin, CSU)

- Average Power

- Peak Power $\sim 20 \mu \mathrm{W} @ 13.9,13.2 \mathrm{~nm} @ 10 \mathrm{~Hz}, 1 \mathrm{~mW} @ 46.9 \mathrm{~nm}$

- Beam divergence

1 - $10 \mathrm{MW}$

$50-100 \mathrm{MW}$

$\sim 0.5$ - 2 mrad (FWHM)

- X-ray laser duration

1 - 8 ps (FWHM)

$100 \mathrm{ps}$

- Longitudinal coherence $\sim 400 \mu \mathrm{m}$ (1/e width)

- Spatial coherence $\quad \sim 3 \%$ for ASE amp. - full coherence for seeded

- Wave front

- Line width

$\lambda 17 @ 32.5$ nm

$\lambda \Delta \lambda=50,000$

- Brightness

$10^{25}-10^{26}$ ph. mm-2 $\mathrm{mrad}^{-2} \mathrm{~s}^{-1}[0.1 \% B W]^{-1}$

$10^{27}$

- Close to transform limited $\Delta E . \Delta t$ operation demonstrated 
Tabletop x-ray lasers are extremely bright, inexpensive compact sources that complement future FELs

\begin{tabular}{|c|c|c|}
\hline \multicolumn{3}{|c|}{$\begin{array}{c}\text { Parameters for collisional } \\
\text { X-ray Laser }\end{array}$} \\
\hline Parameters & $\begin{array}{l}\text { GRIP } \\
\text { XRL }\end{array}$ & $\begin{array}{l}\text { COMET } \\
\text { XRL }\end{array}$ \\
\hline $\begin{array}{l}\text { Pump }(\mathrm{J}): \\
\text { XRL }(\mathrm{J}): \\
\text { Photons: } \\
\text { Rate }(\mathrm{Hz}): \\
\lambda(\mathrm{nm}): \\
\text { Source }\left(\mu \mathrm{m}^{2}\right): \\
\text { Div. }\left(\mathrm{mrad}^{2}\right): \\
\text { Pulse }(\mathrm{ps}): \\
\text { Peak } \mathrm{B}^{\star}: \\
\text { Average } \mathrm{B}^{\star} \neq:\end{array}$ & $\begin{array}{c}150 \mathrm{~mJ} \\
10 \mathrm{~nJ} \\
10^{9} \\
10 \\
18.9 \\
9 \times 20 \\
3 \times 5 \\
2 \\
2.0 \times 10^{23} \\
3.7 \times 10^{12}\end{array}$ & $\begin{array}{c}5 \mathrm{~J} \\
>10 \mu \mathrm{J} \\
10^{12} \\
0.004 \\
12-47 \\
25 \times 100 \\
2.5 \times 6 \\
2-8 \\
1.6 \times 10^{25} \\
1.3 \times 10^{11}\end{array}$ \\
\hline \multicolumn{3}{|c|}{$\begin{array}{l}\text { * }\left[\mathrm{Ph} . \mathrm{mm}^{-2} \mathrm{mrad}^{-2} \mathrm{~s}^{-1}(0.1 \% \mathrm{BW})\right)^{-1} \\
\text { ‡ For } 10 \mathrm{~Hz} \text { operation }\end{array}$} \\
\hline
\end{tabular}

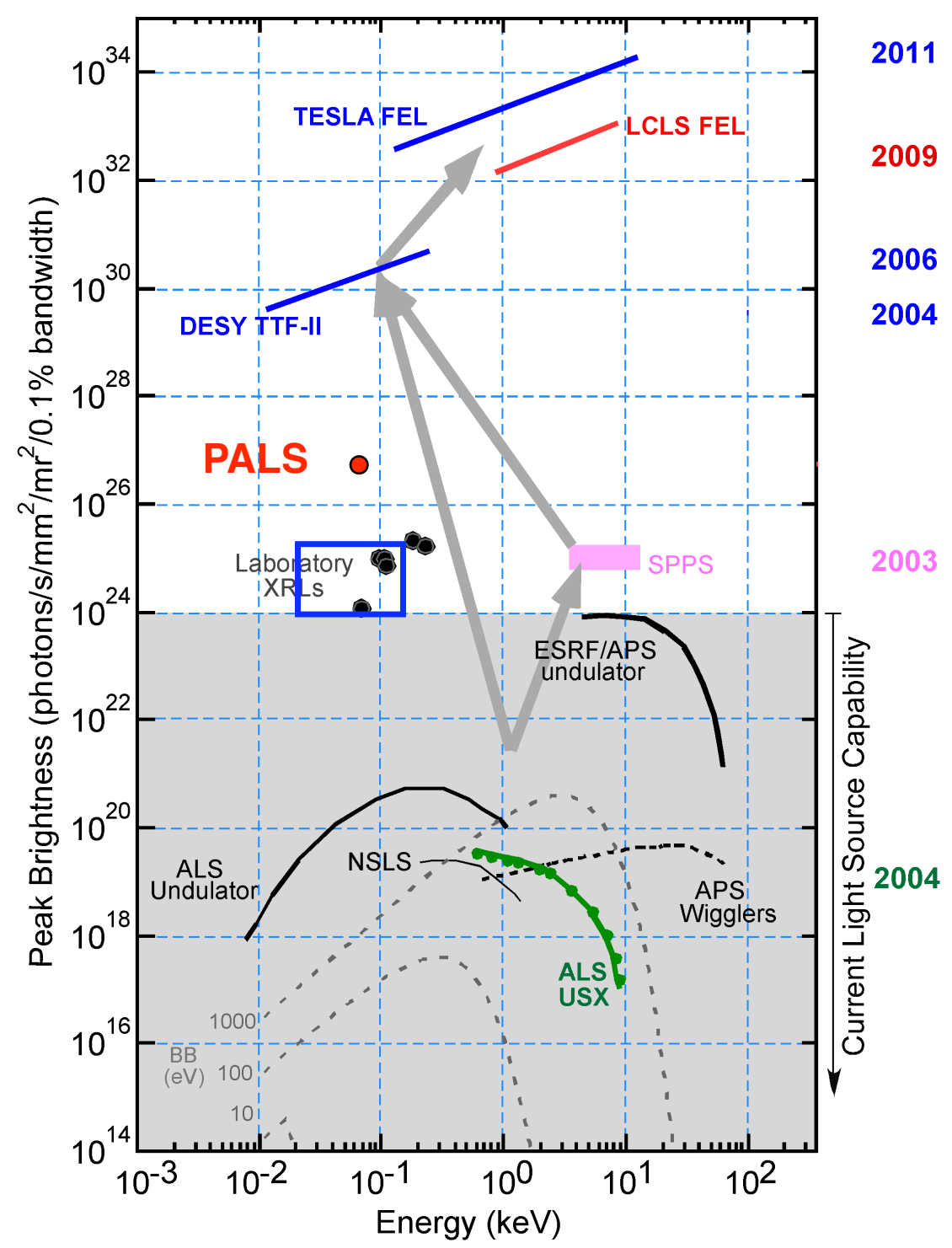




\section{Recent developments in X-ray Laser Sources}

- Grazing Incidence Pumping (GRIP)

- Higher Order Harmonic Seeding into OFI amplifier

- Higher Order Harmonic Seeding into GRIP plasma amplifier 


\section{Grazing Incidence Pumping (GRIP) x-ray laser produced by absorbing pump energy efficiently in gain region: $10 \mathrm{~Hz} X R L$}
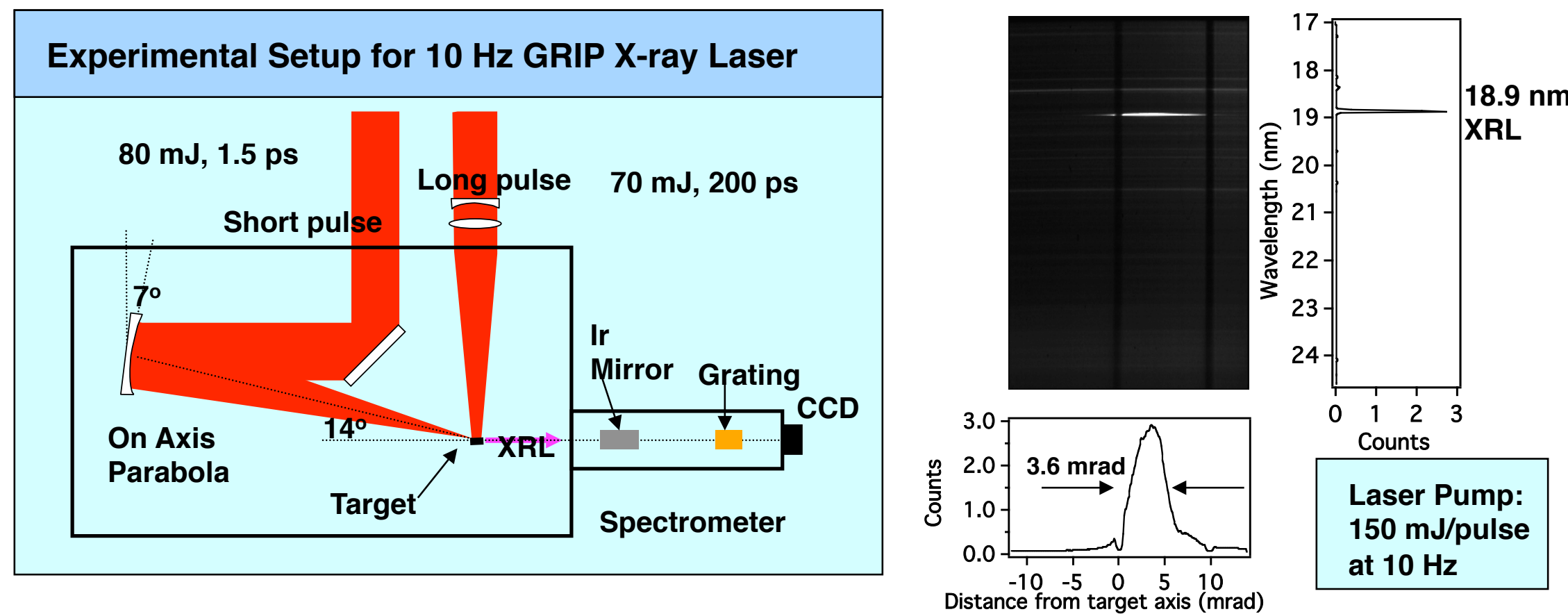

-Short pulse propagates in plasma up to a specific electron density

-Short pulse is then refracted back into gain region

-Short pulse angle given by $\theta=\sqrt{n_{e 0} / n_{e c}}$ where $\mathbf{n}_{\mathbf{e} 0}=$ density at turning point

-Traveling wave pump inherent and no restriction on target length

-Absorption efficiency in gain region increases to $50 \%$ for GRIP 


\section{Lasing observed at wavelengths as short as $10.9 \mathrm{~nm}$}

\section{Colorado}

State

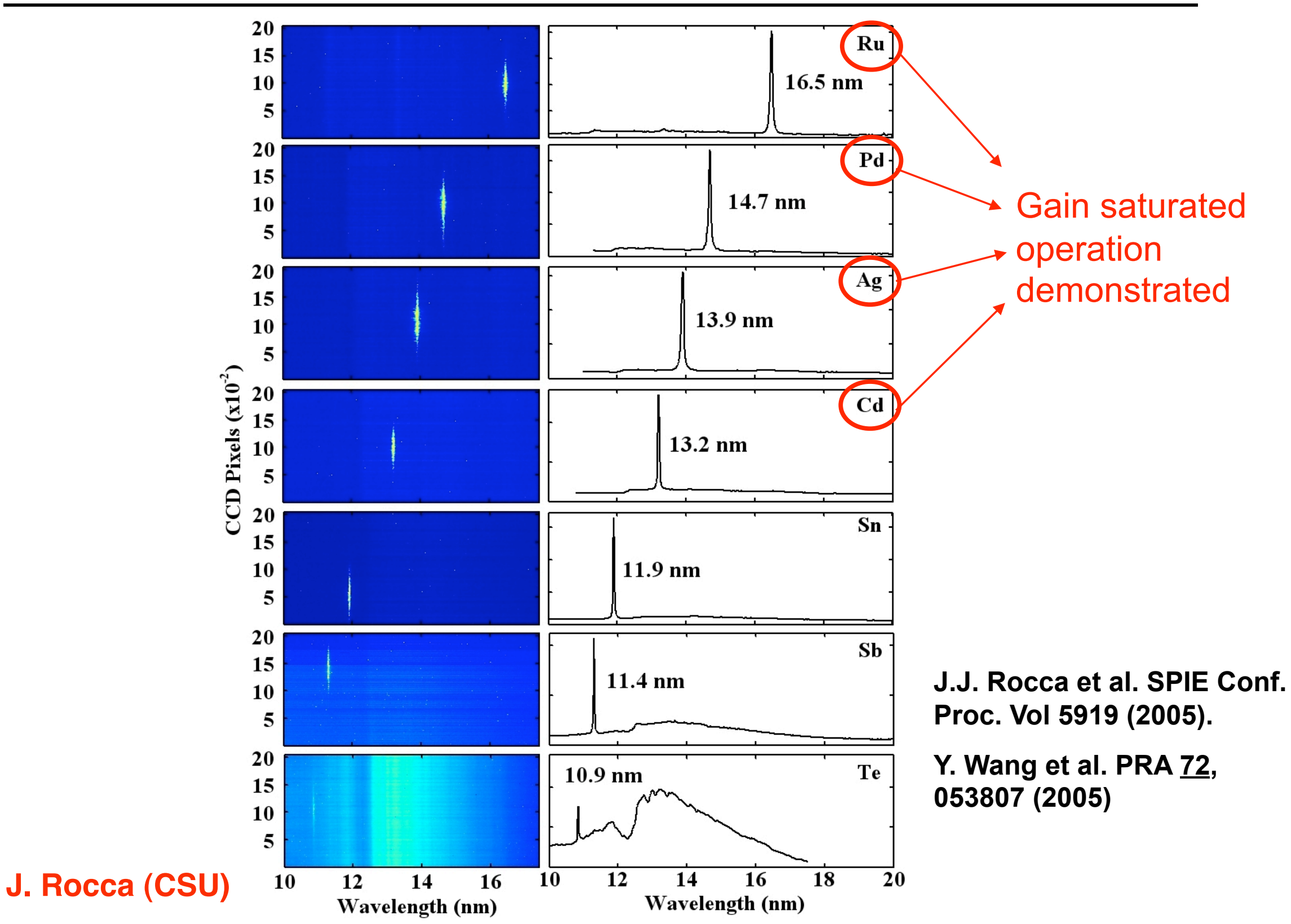


Gain-saturated Ni-like $13.2 \mathrm{~nm}$ Ni-like Cd laser

\section{$1 \mathrm{~J}$ short pulse - 23 degrees grazing incidence angle}
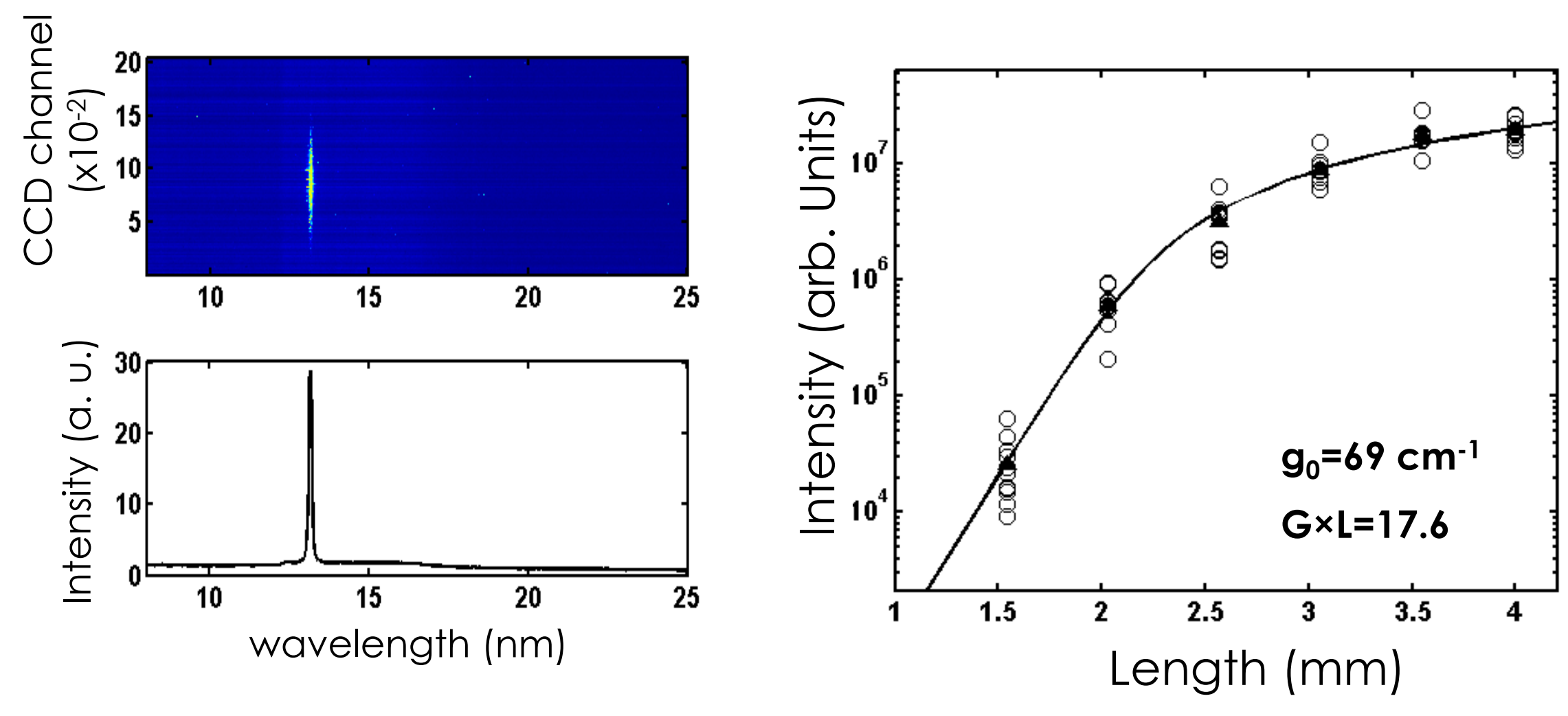

J.J. Rocca, Opt. Lett. 30, 2851 (2005). 


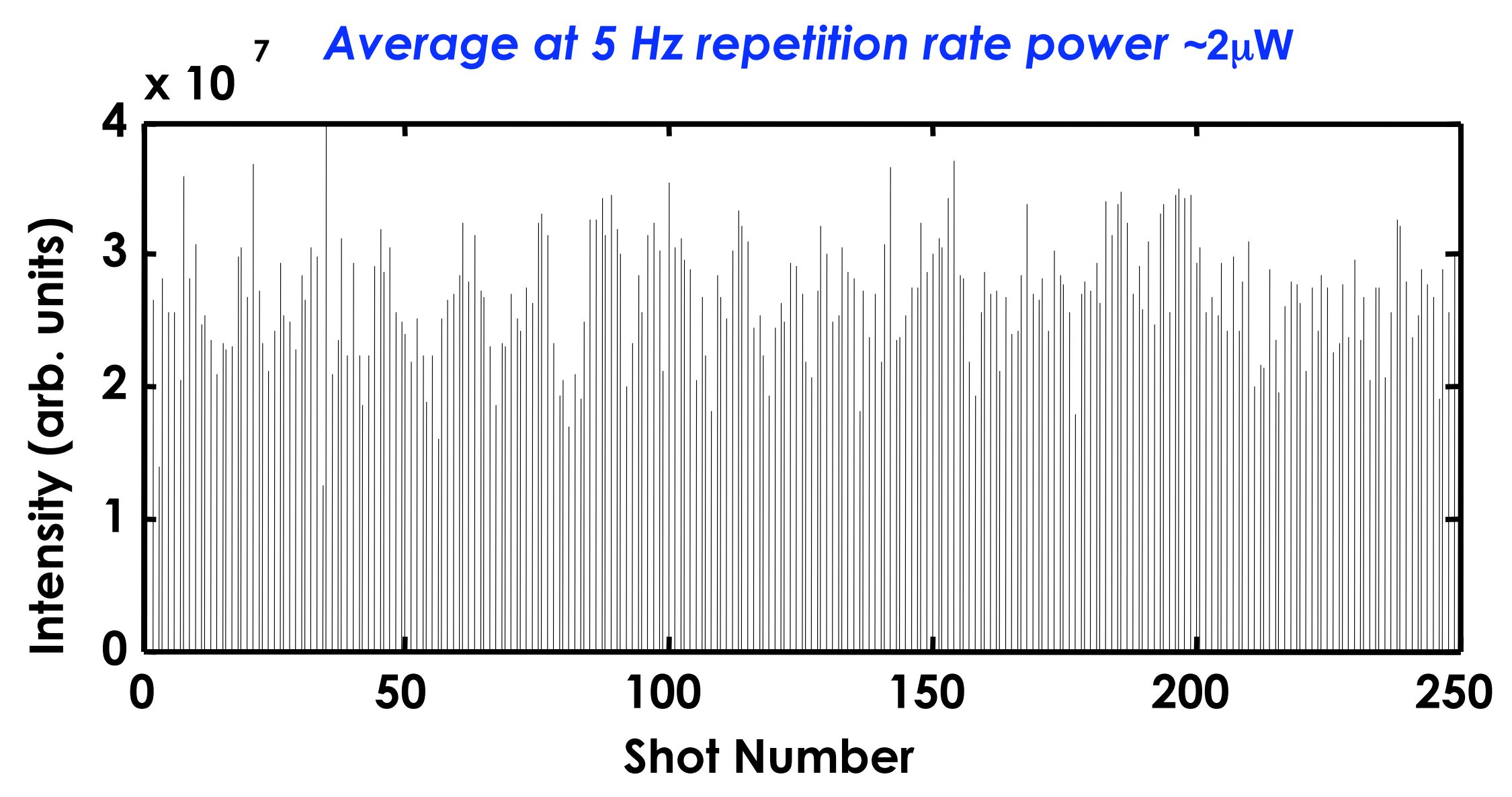

Similar performance also obtained for Ni-like Cd J. Rocca (CSU)

@ $13.2 \mathrm{~nm},>1 \mu \mathrm{W}$ average power 
Gain-Saturated output of table-top Laser extended to $10.9 \mathrm{~nm}$ at $1 \mathrm{~Hz}$ rep. rate

\section{Nickel-like Tellurium}
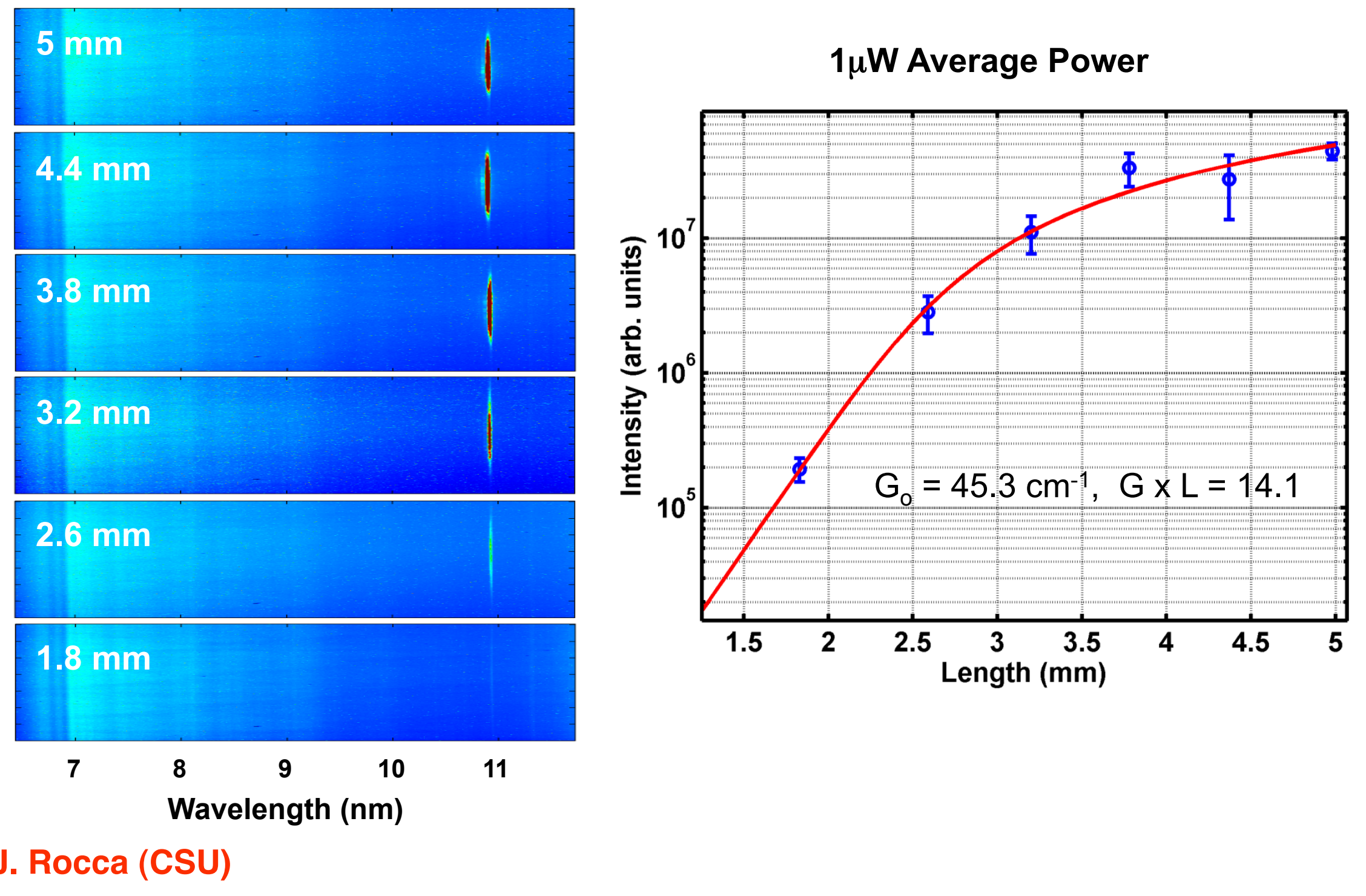


\section{Coupling one harmonic to an XRL amplifier : set up}

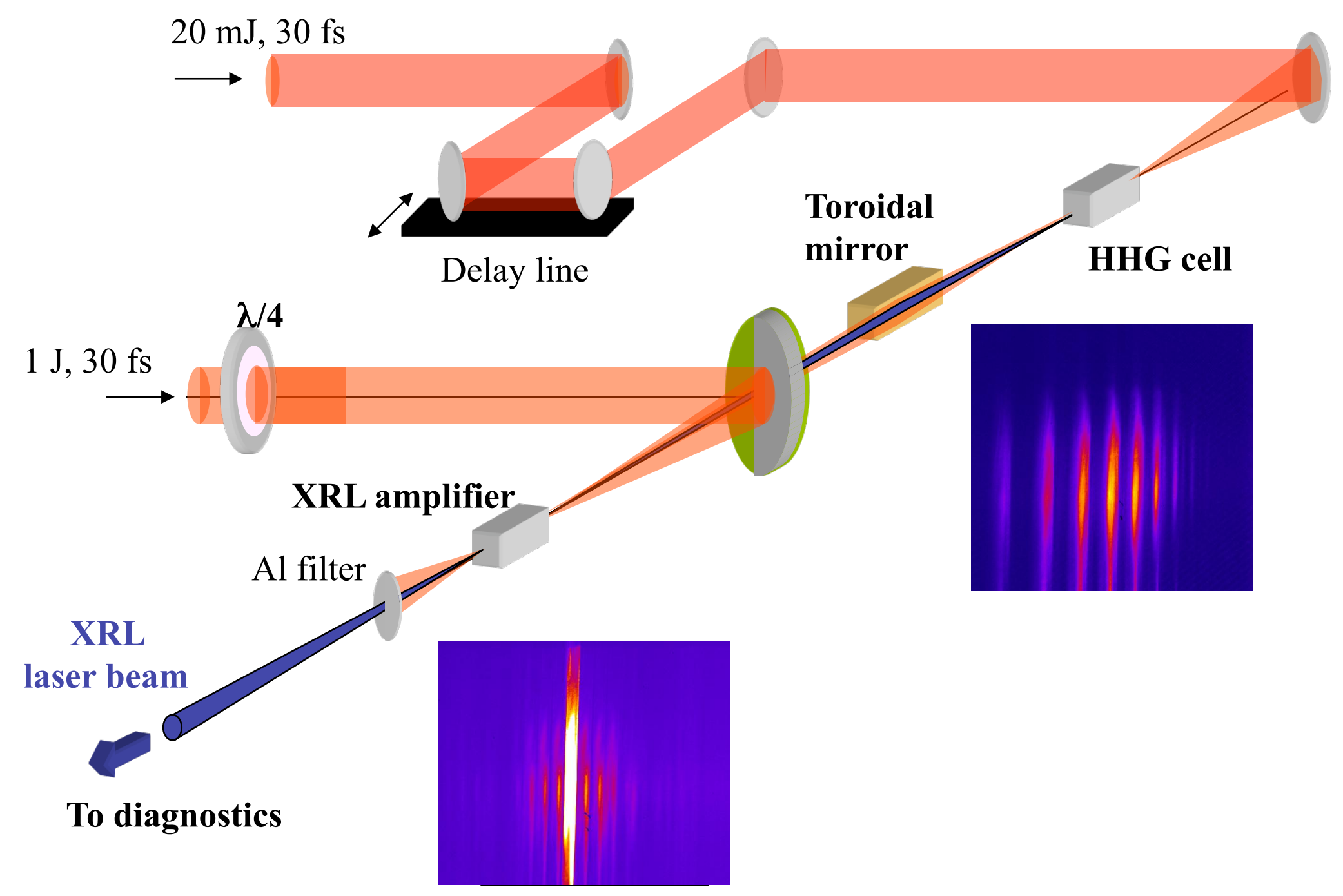

S. Sebban (LOA) 


\section{Evidence of amplification of the $32.8 \mathrm{~nm} \mathrm{Kr}$ IX laser}

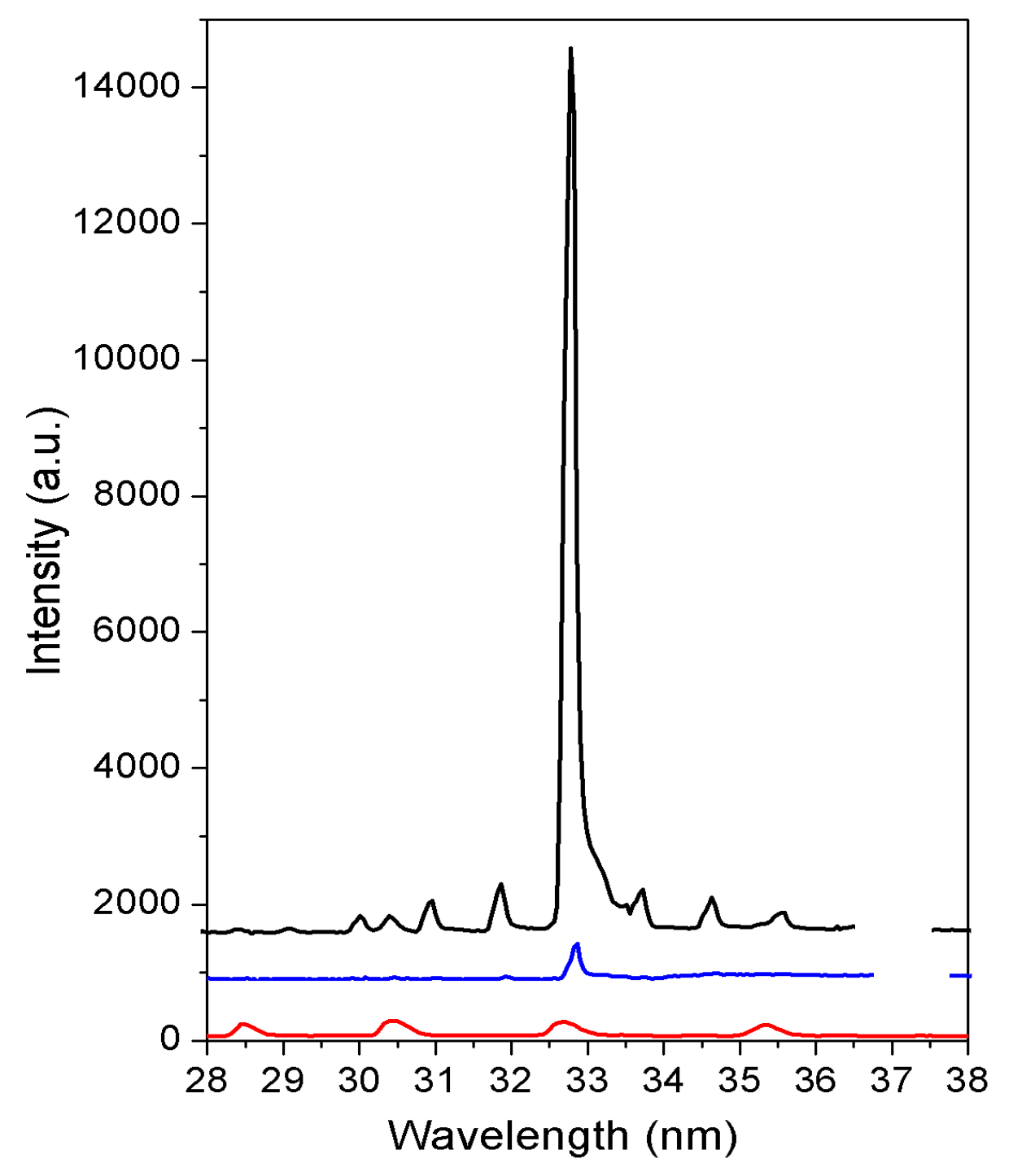

- SSXRL

— ASE emission

— HHG seed

S. Sebban (LOA) 


\section{Far field pattern of the $32.8 \mathrm{~nm}$ seeded $\mathrm{x}$-ray laser}

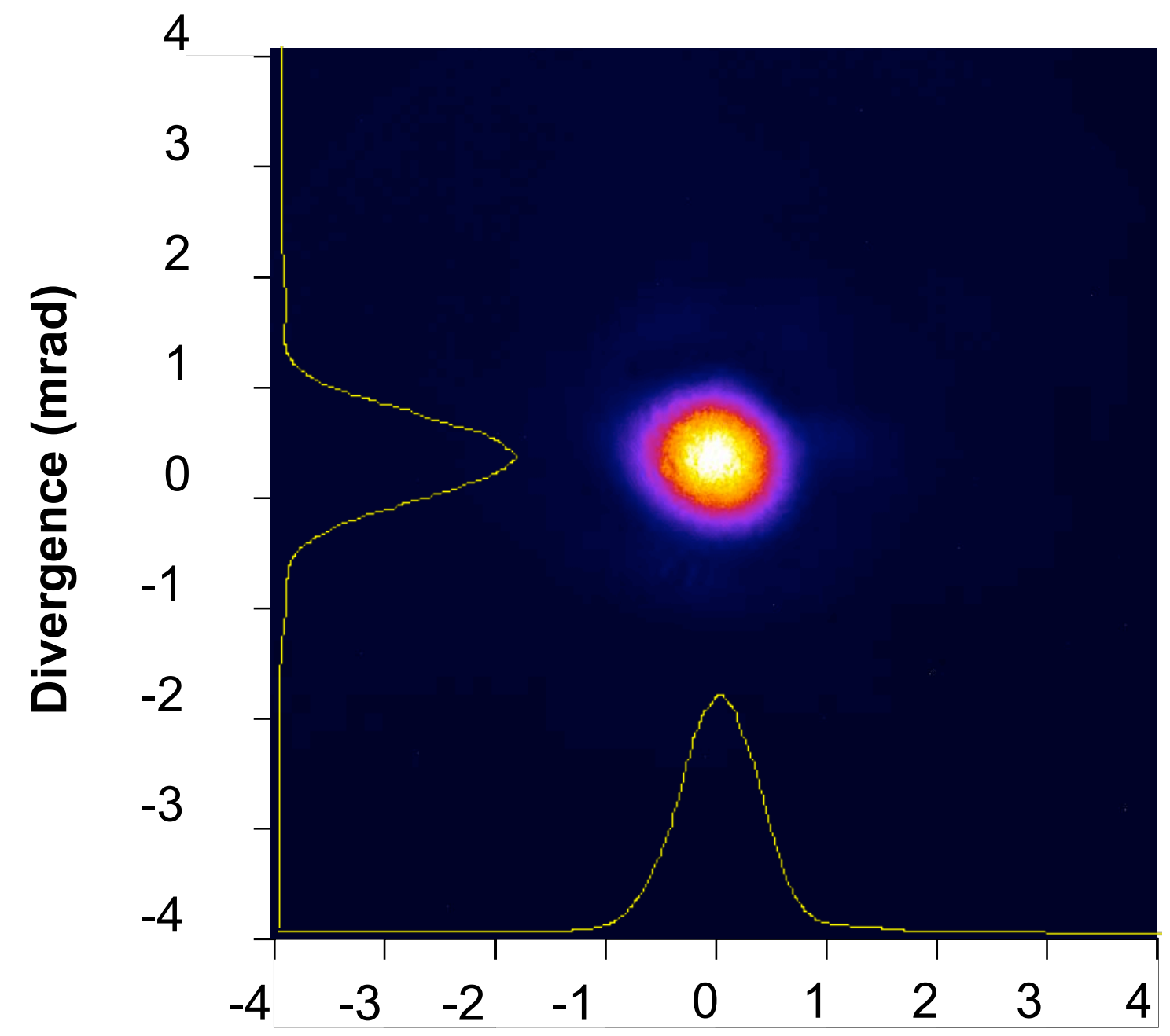

$\mathrm{E}=0.7 \mu \mathrm{J}$ per shot

Divergence : $0.7 \mathrm{mrad}$

S. Sebban (LOA) 


\section{Transverse coherence : \\ Young's double slit experiment}

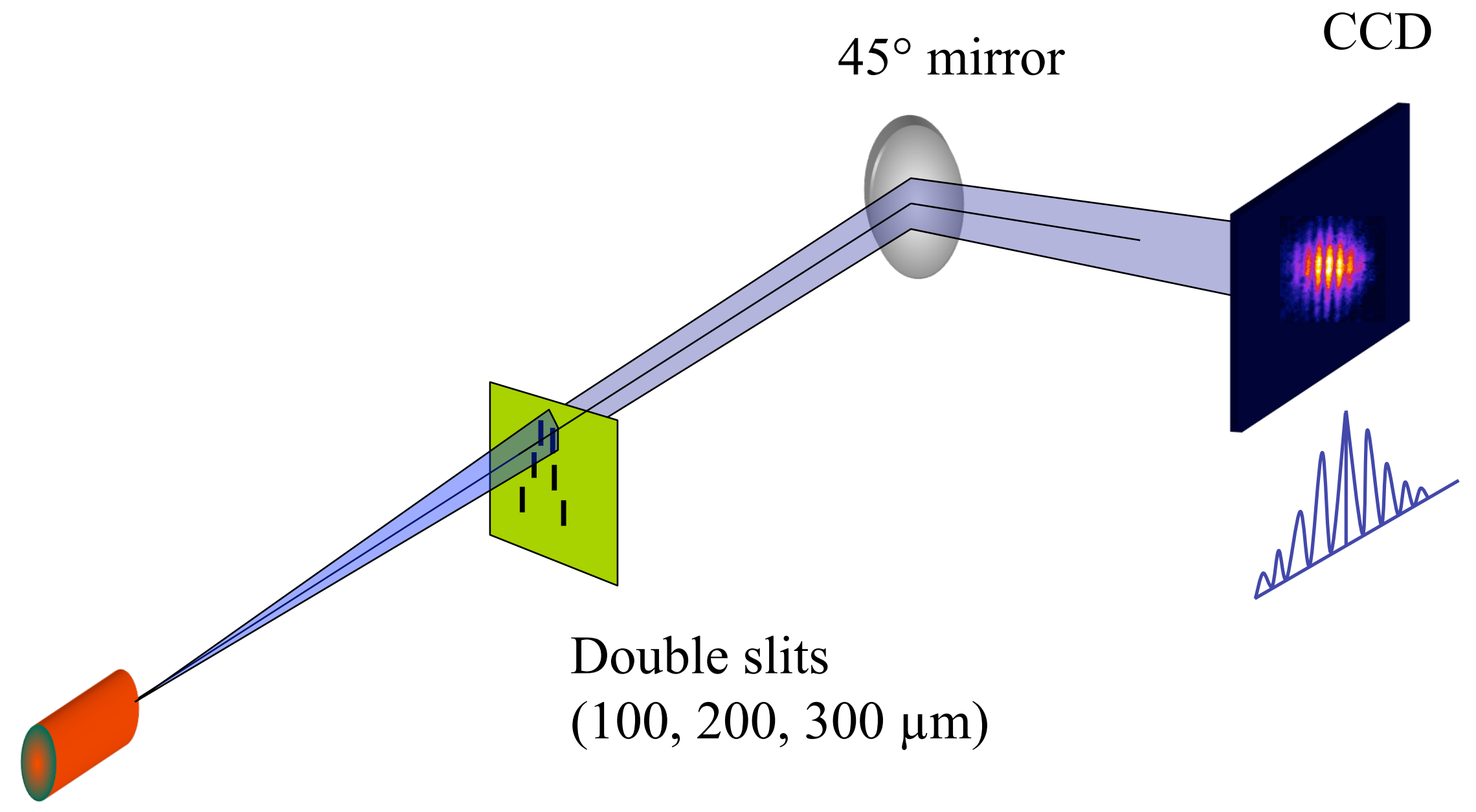

XRL plasma 


\section{High spatial coherence of the seeded soft XRL}

\section{(Young' slit experiment)}

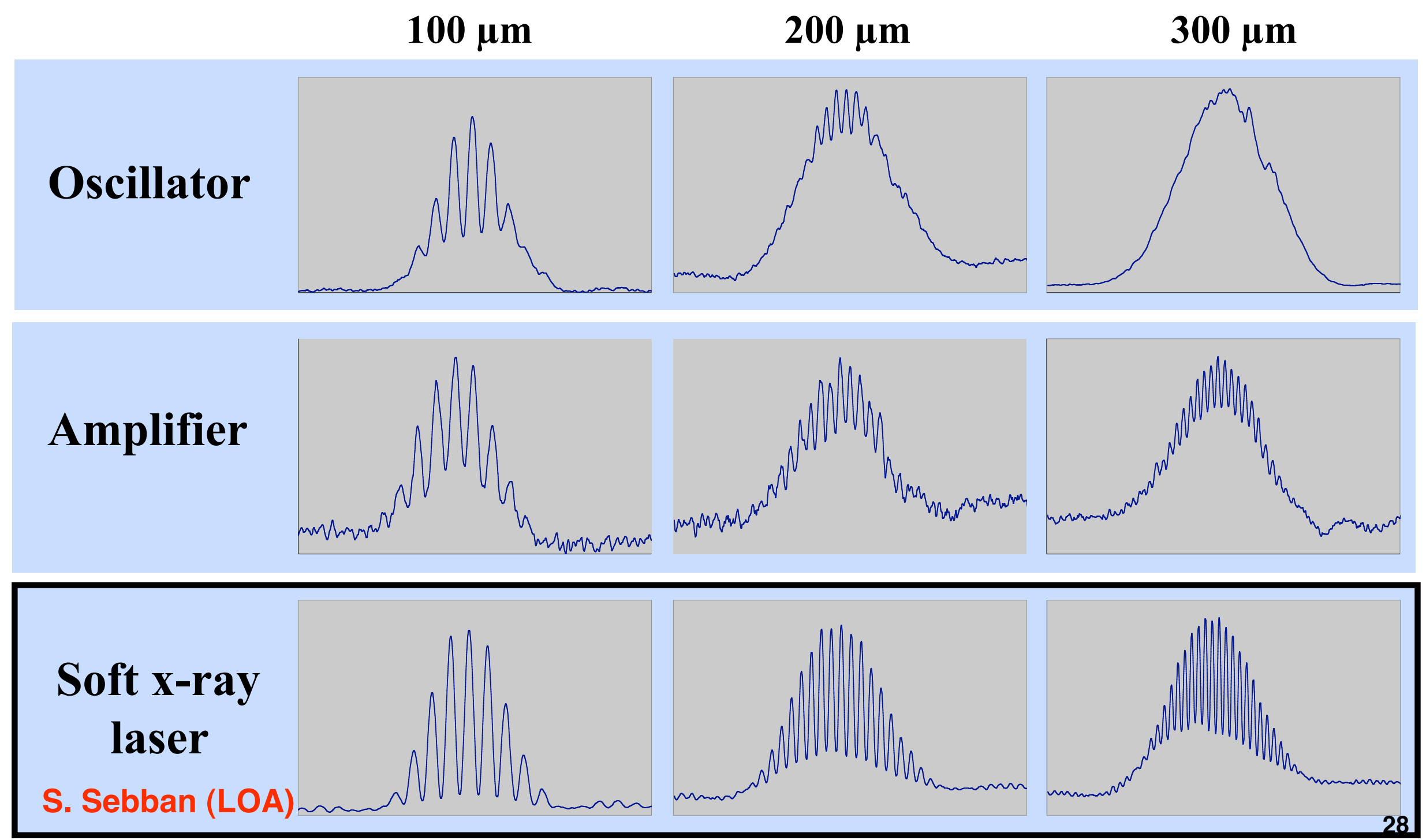




\section{Contrast of the fringes pattern (Young' slit experiment)}
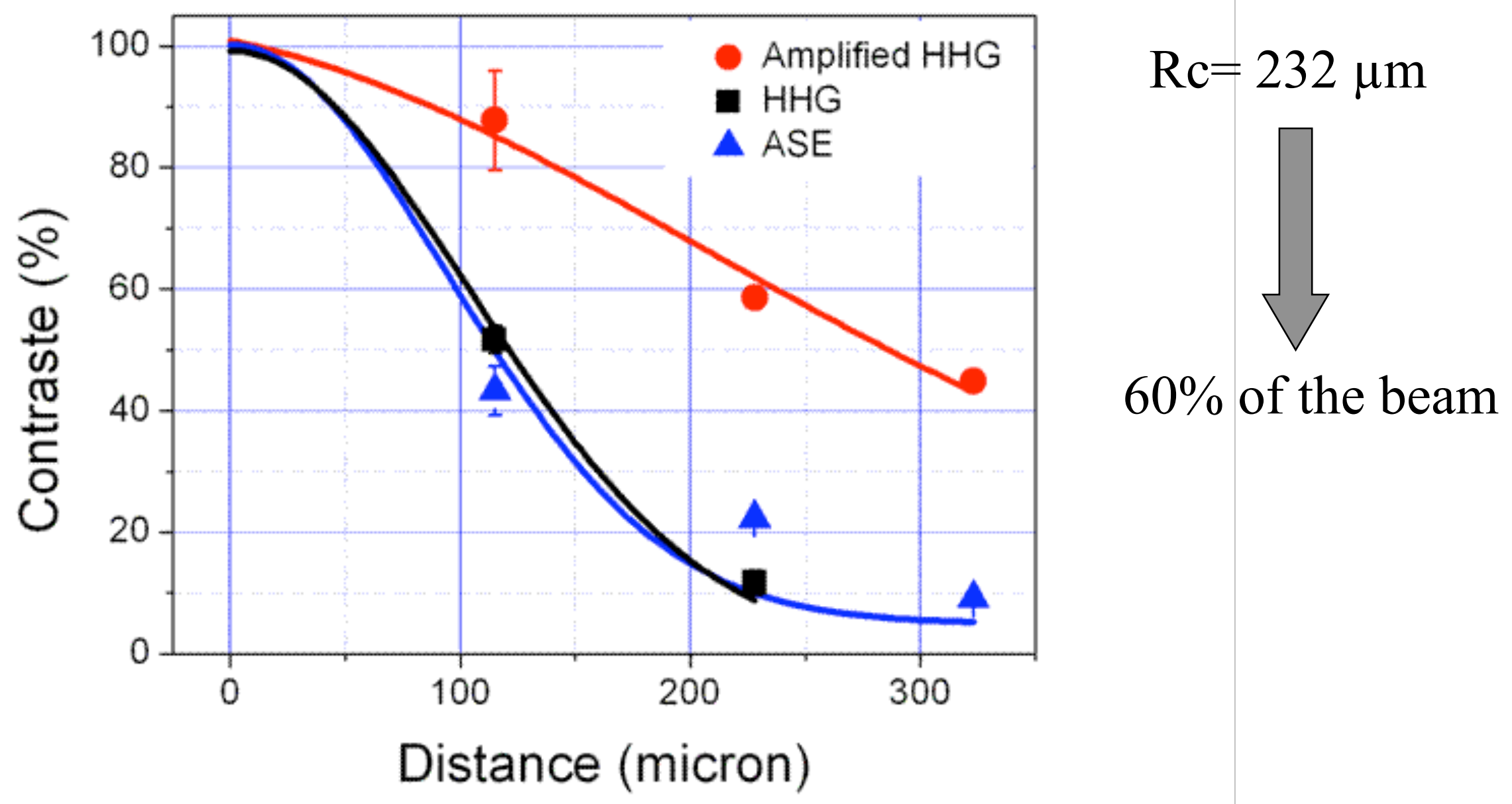

J. Ph. Goddet et al., Opt. Lett., 32, 1498 (2007) 


\section{Seeding with high harmonic pulse can greatly increase coherence and beam brightness}

- Decreased divergence

- Shorter pulsewidth

- Defined polarization

- Higher brightness

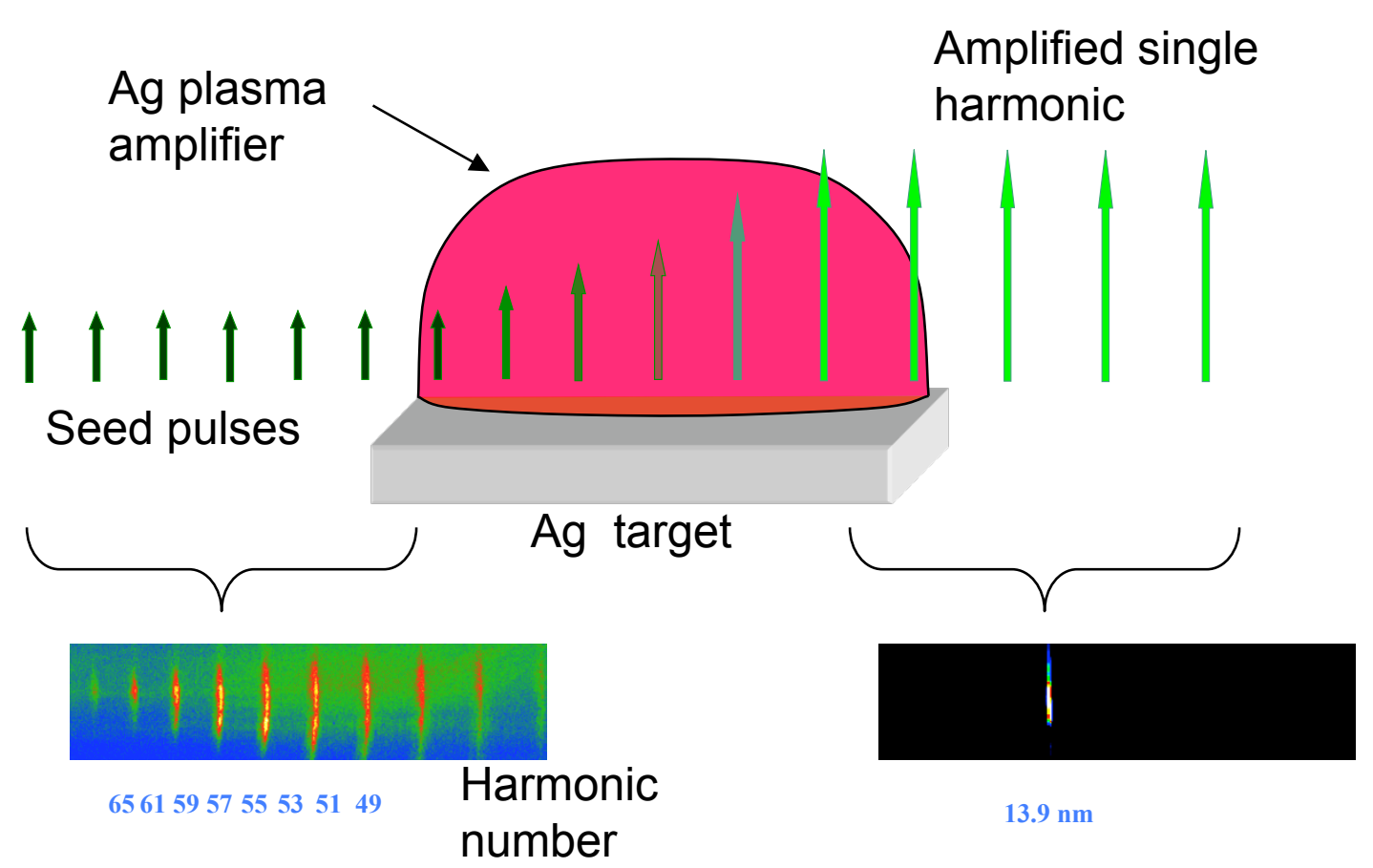

- $\quad$ Proof of principle experiment: T. Ditmire et al. Phys. Rev. A. 51, R 4337, (1995): Amplification of HHG by $\sim 3 X$ in the $\lambda=25.1 \mathrm{~nm}$ line of a Gallium laser amplifier

- HHG seeding of OFI amplifier: P. Zeitoun, G. Faivre, S. Sebban, T. Mocek et al, Nature , 431, 426, (2004).

- Injection- seeded soft X-ray laser using solid target plasmas: Y.Wang, E.Granados, M. Larotonda, M. Berrill, B.Luther, D. Patel, C.S. Menoni and J.J. Rocca, Phys..Rev.Lett, $97,123901(2006)$

J. Rocca (CSU) 
Demonstration of injection-seeded EUV laser in dense amplifier created by irradiation of solid target

\section{Colorado} State
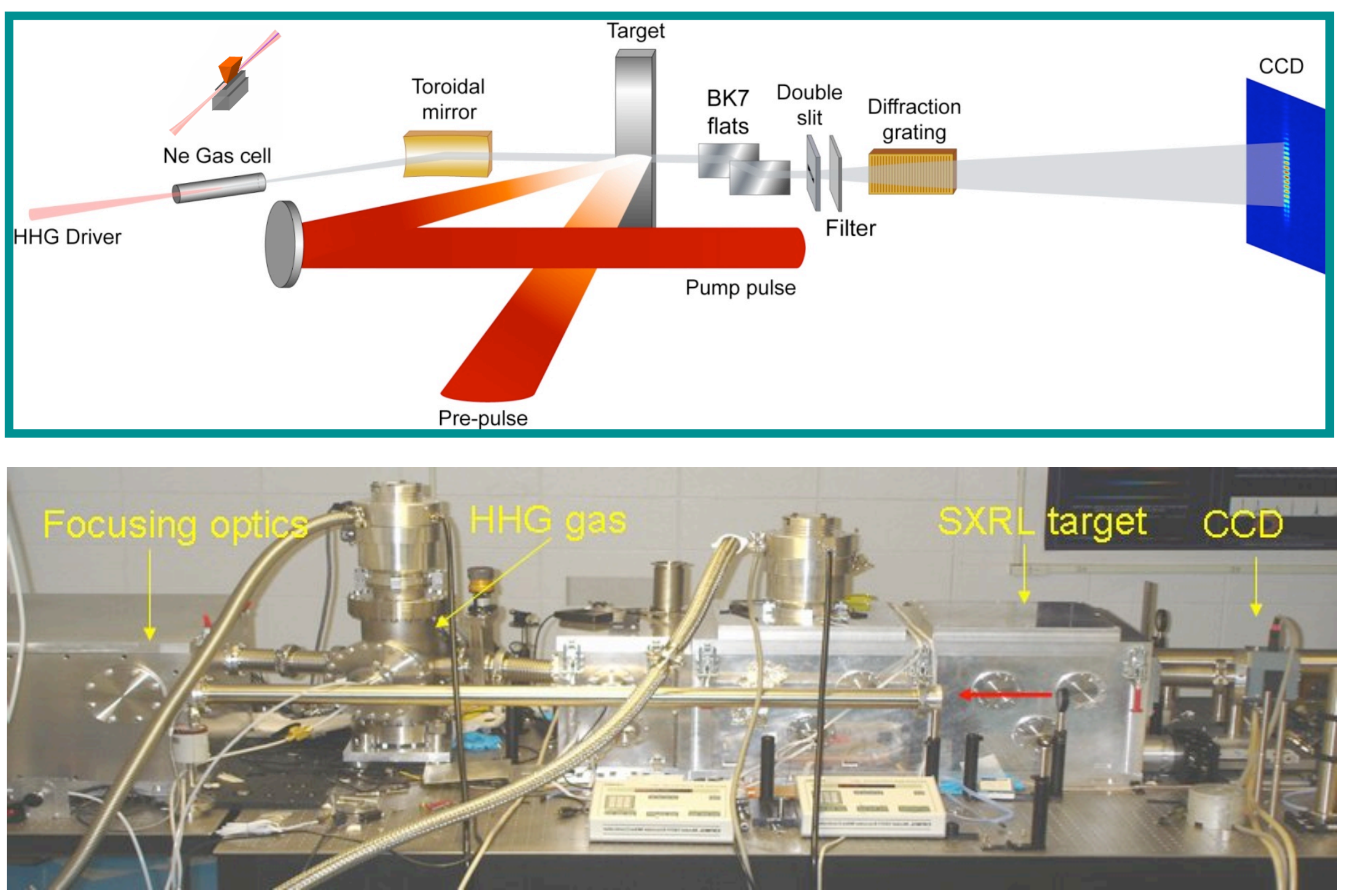

J. Rocca (CSU) 


\section{Simulation of High Harmonic seeded $32.6 \mathrm{~nm}$ $\mathrm{Ne}$-like Ti amplifier}

Colorado

$32.6 \mathrm{~nm}$ Ne-like Ti amplifier seeded by $25^{\text {th }}$ harmonic of Ti:Sapphire laser

Bandwidth narrowing of amplified seed pulse
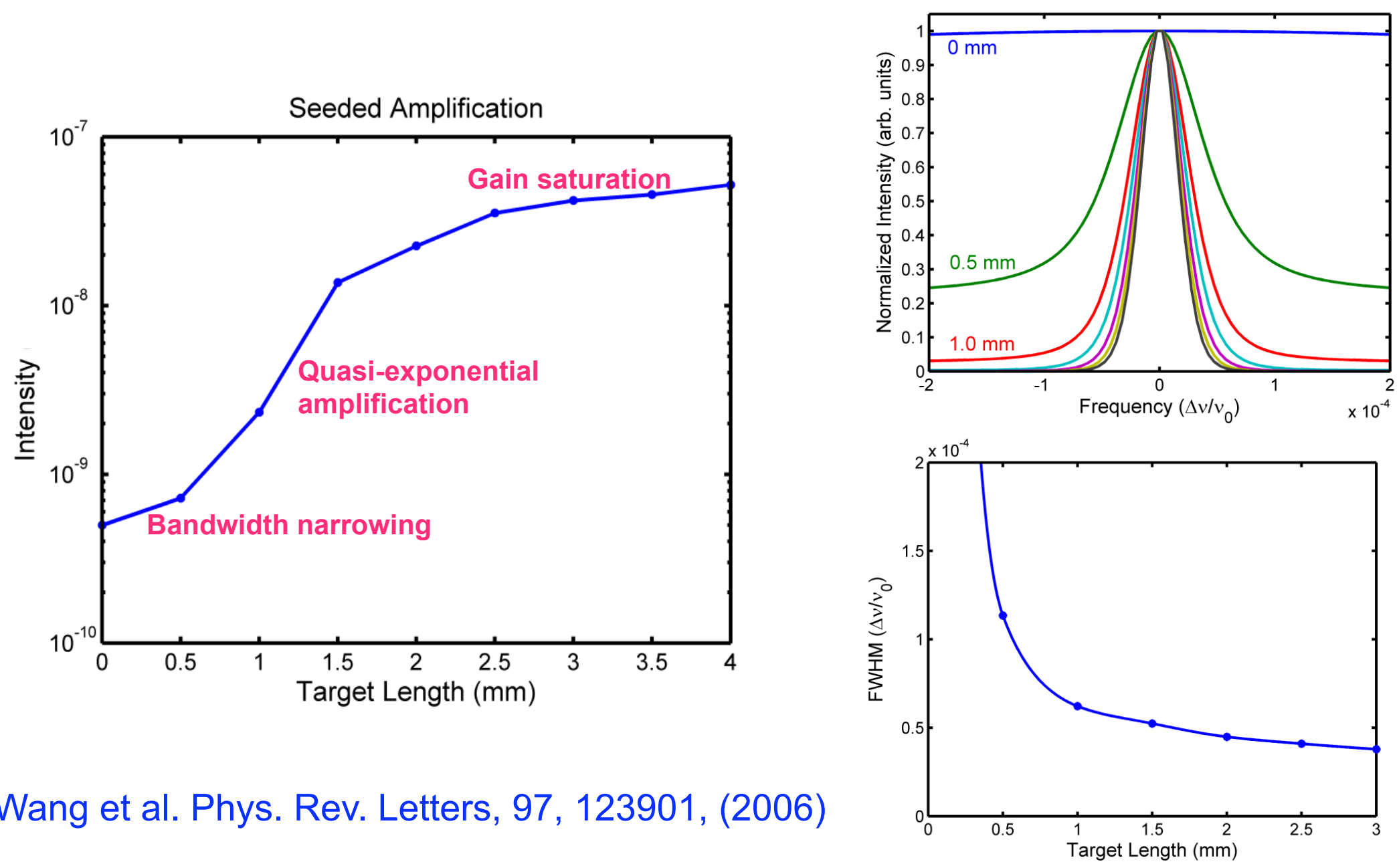

Y. Wang et al. Phys. Rev. Letters, 97, 123901, (2006)

J. Rocca (CSU) 


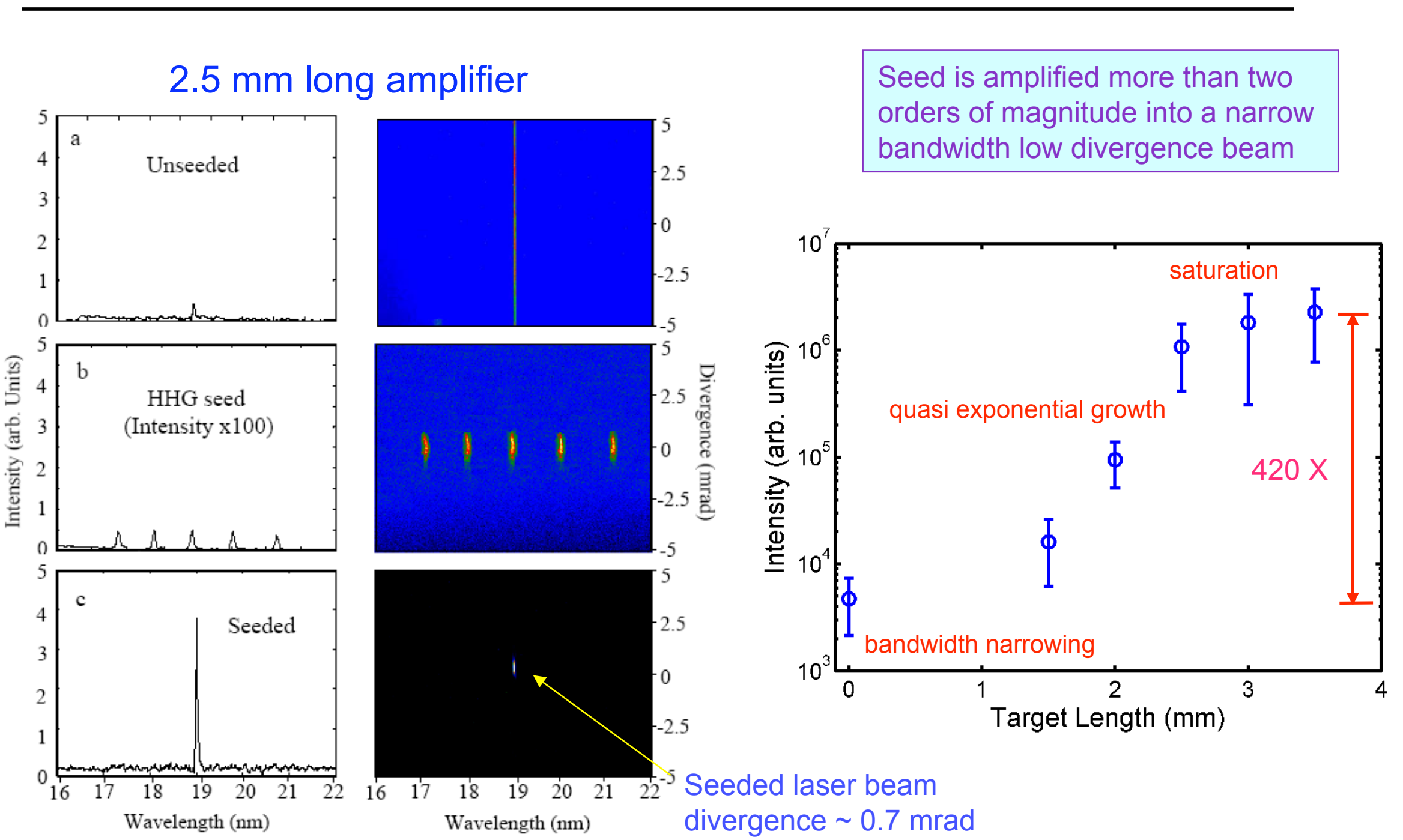

Y. Wang et al. Nature Photonics, 2, 94, (2008)

J. Rocca (CSU) 


\section{Essentially fully coherent output Ni-like Mo 18.9 nm Colopado \\ State}
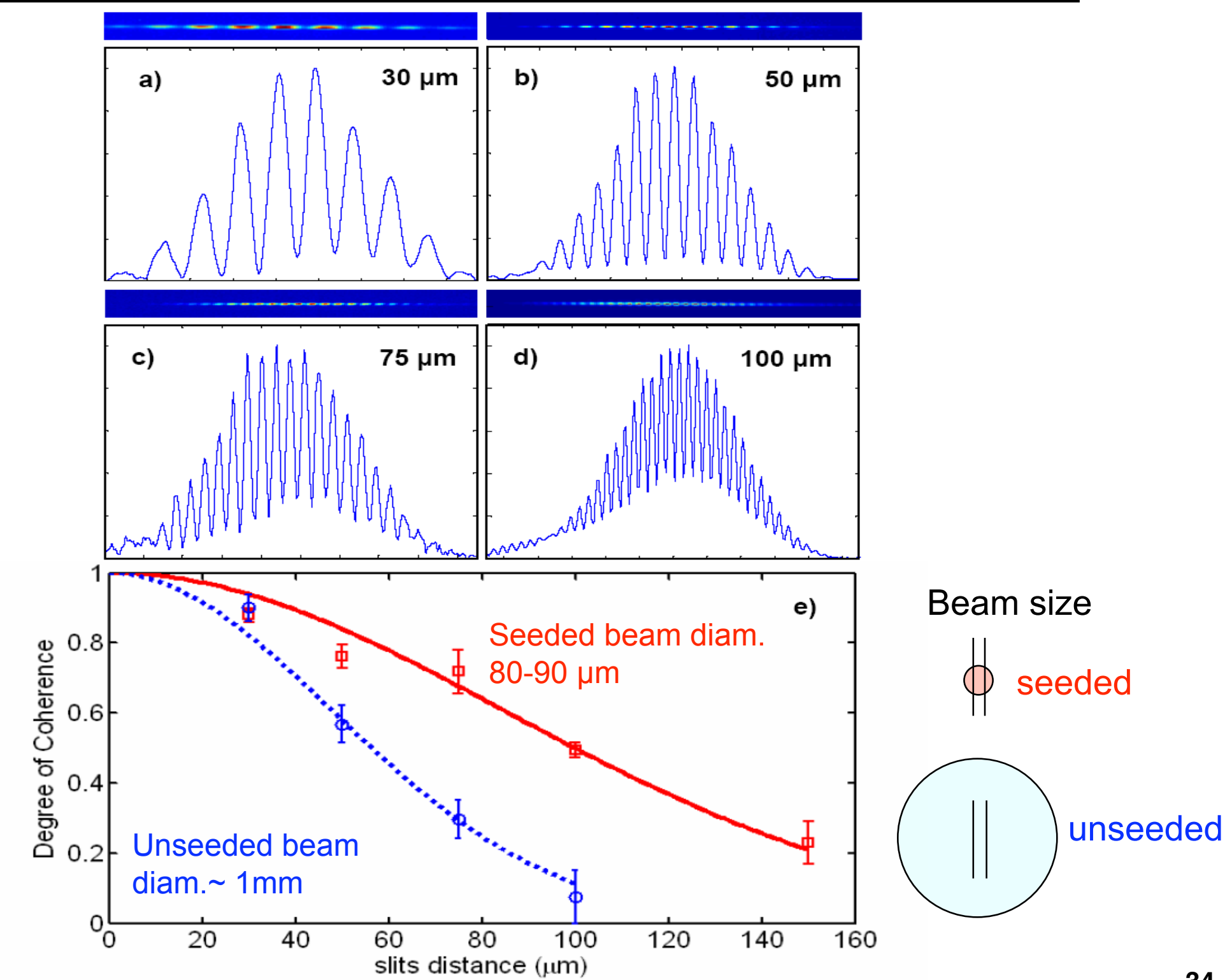

Beam size

$\oiint$ seeded

J. Rocca (CSU) 


\section{$\underline{\text { X-ray Laser Applications }}$}

- X-ray Laser Interferometry 


\section{X-ray Laser Interferometry with grating beam splitters first demonstrated at $46.9 \mathrm{~nm}$ at CSU, extended to $14.7 \mathrm{~nm}$}
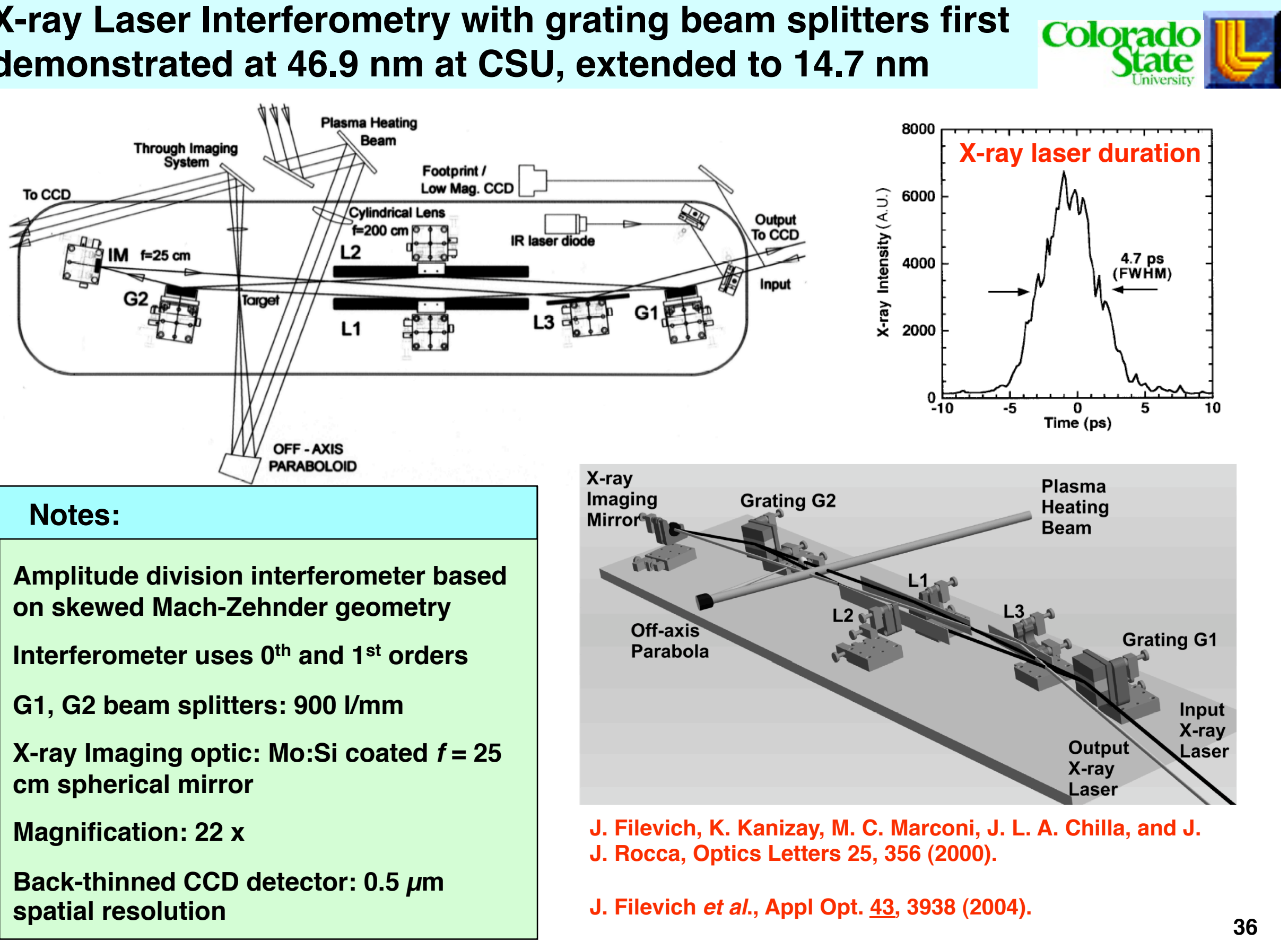

J. Filevich, K. Kanizay, M. C. Marconi, J. L. A. Chilla, and J.

J. Rocca, Optics Letters 25, 356 (2000).

J. Filevich et al., Appl Opt. 누, 3938 (2004). 
Sequence of ps interferograms shows formation of remarkable dynamic plasma features at all times

Flat Al targets heated by $3 \mathrm{~J}, 12 \mu \mathrm{m}$ wide, 600 ps pulse at $>10^{13} \mathrm{~W} \mathrm{~cm}^{-2}$
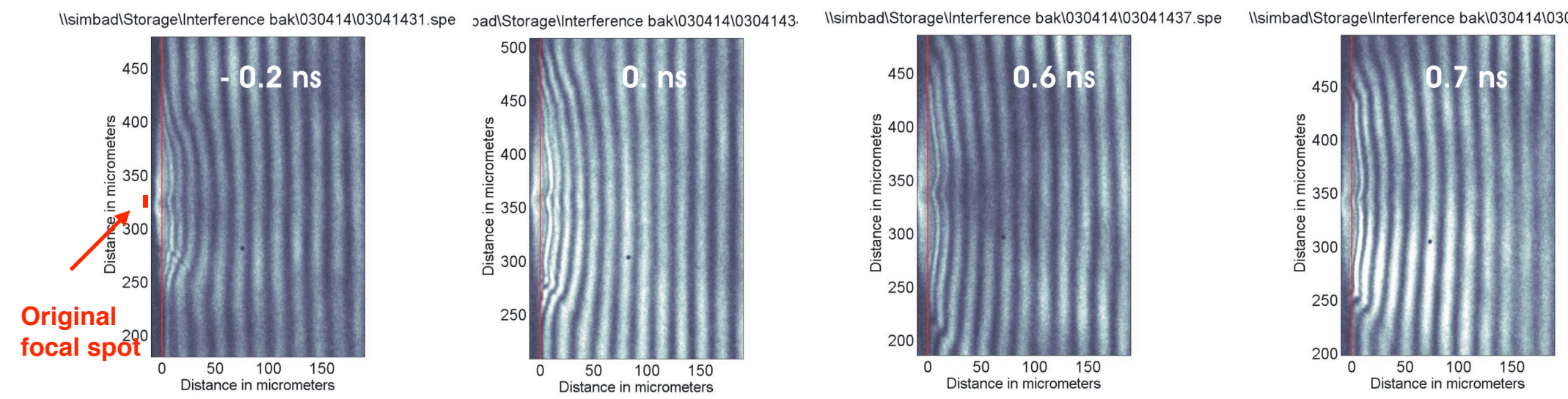

- 2-D lateral expansion observed early on - plasma pressure gradients

- On-axis dip formed due to instability - high local $T_{e}$, focal spot geometry IIsimbadIStoragellnterference bak10304141030414 ilsimbadlStoragellnterference bak10304091030409
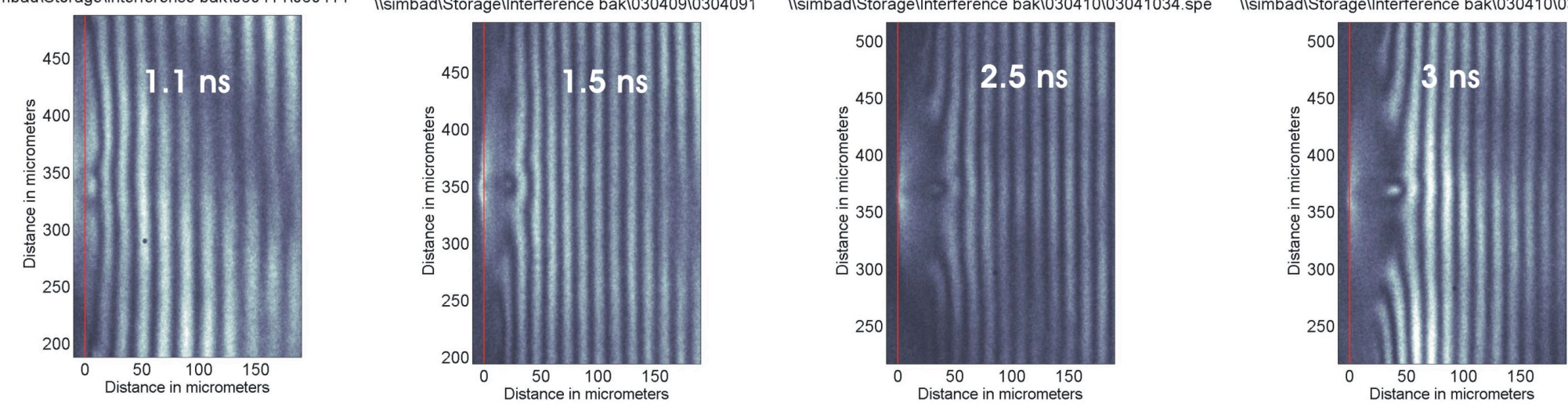

- Fringe reversal at late time due to bound electrons in $\mathrm{Al}-\mathrm{Al}^{3+}$ ions 


\section{Experiments used to benchmark 2-D LASNEX for high energy density laser-produced plasmas - real tool}

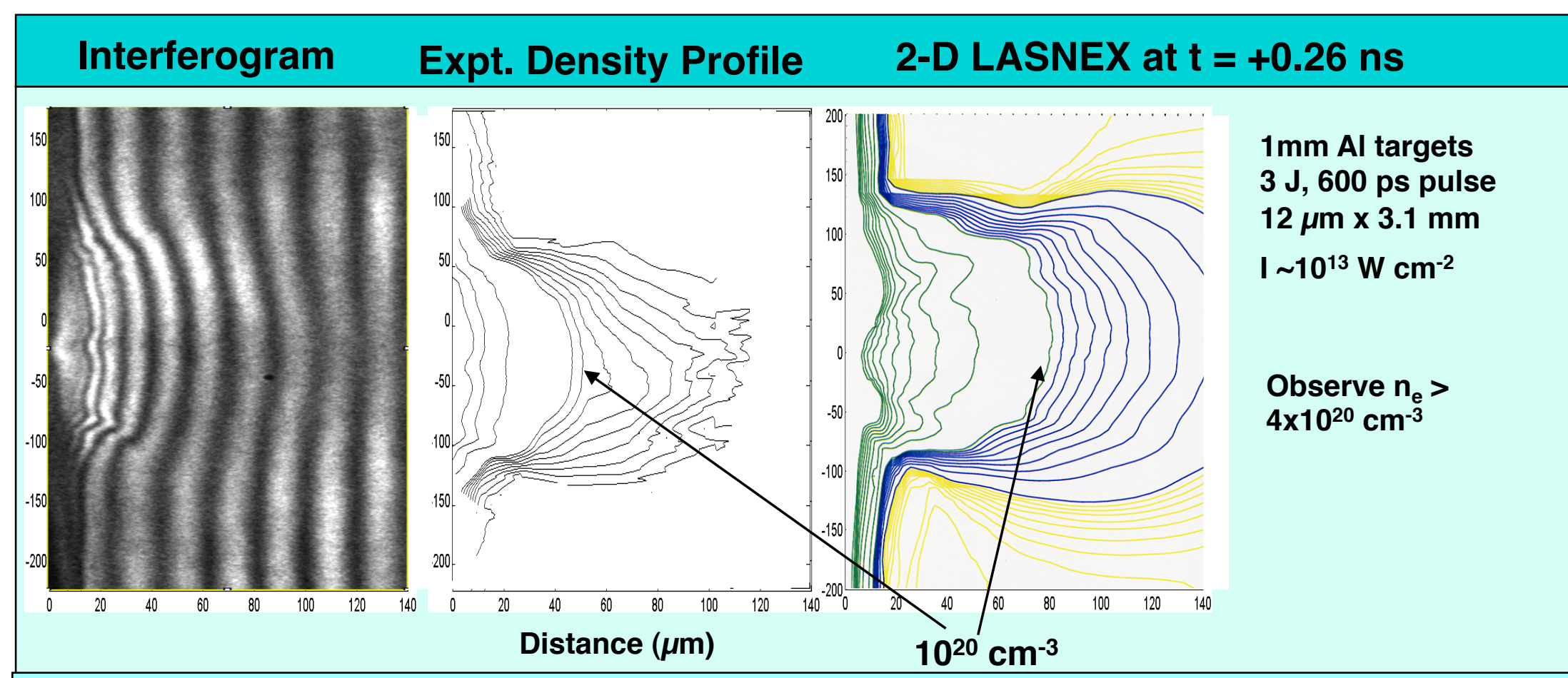

- Small $12 \mu \mathrm{m}$ width results in substantial 2D plasma expansion - reduced on-axis density

- 1D and 1.5D LASNEX simulations do not accurately model plasma conditions

- 2D simulations use experimental focal spot and temporal pulse shape

- Plasma pressure gradients, radiative heating and thermal conduction produces side lobes

Short wavelength, $\sim 1 \mu \mathrm{m}$ spatial and ps time resolution essential 
IV. Semi-cylindrical target: Experimental setup at CSU for probing laser-heated half-hohlraum type geometry

\section{Laser Irradiation conditions:}

- $10^{12} \mathrm{Wcm}^{-2}, 600 \mathrm{~mJ}$ at $800 \mathrm{~nm}$ wavelength, 120 ps (FWHM)

- Laser focus: $300 \mu \mathrm{m} \times 1.5 \mathrm{~mm}$ line

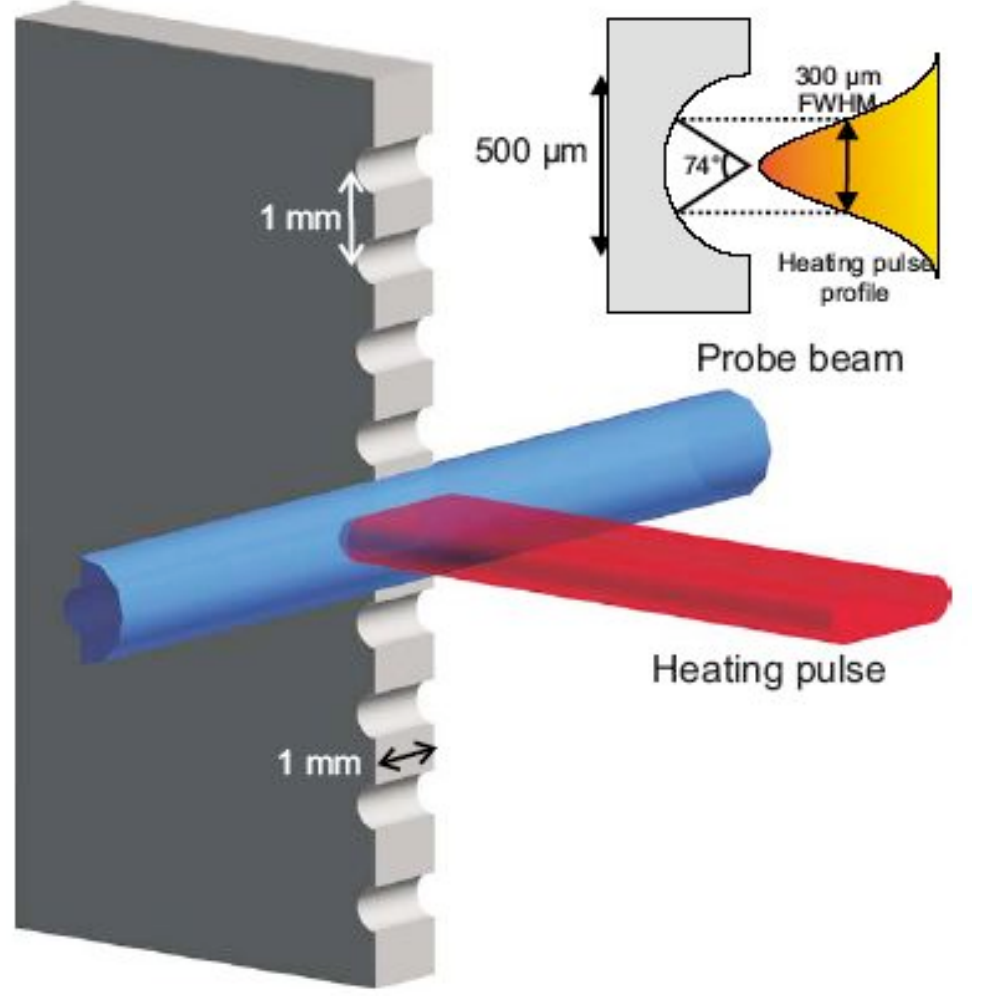

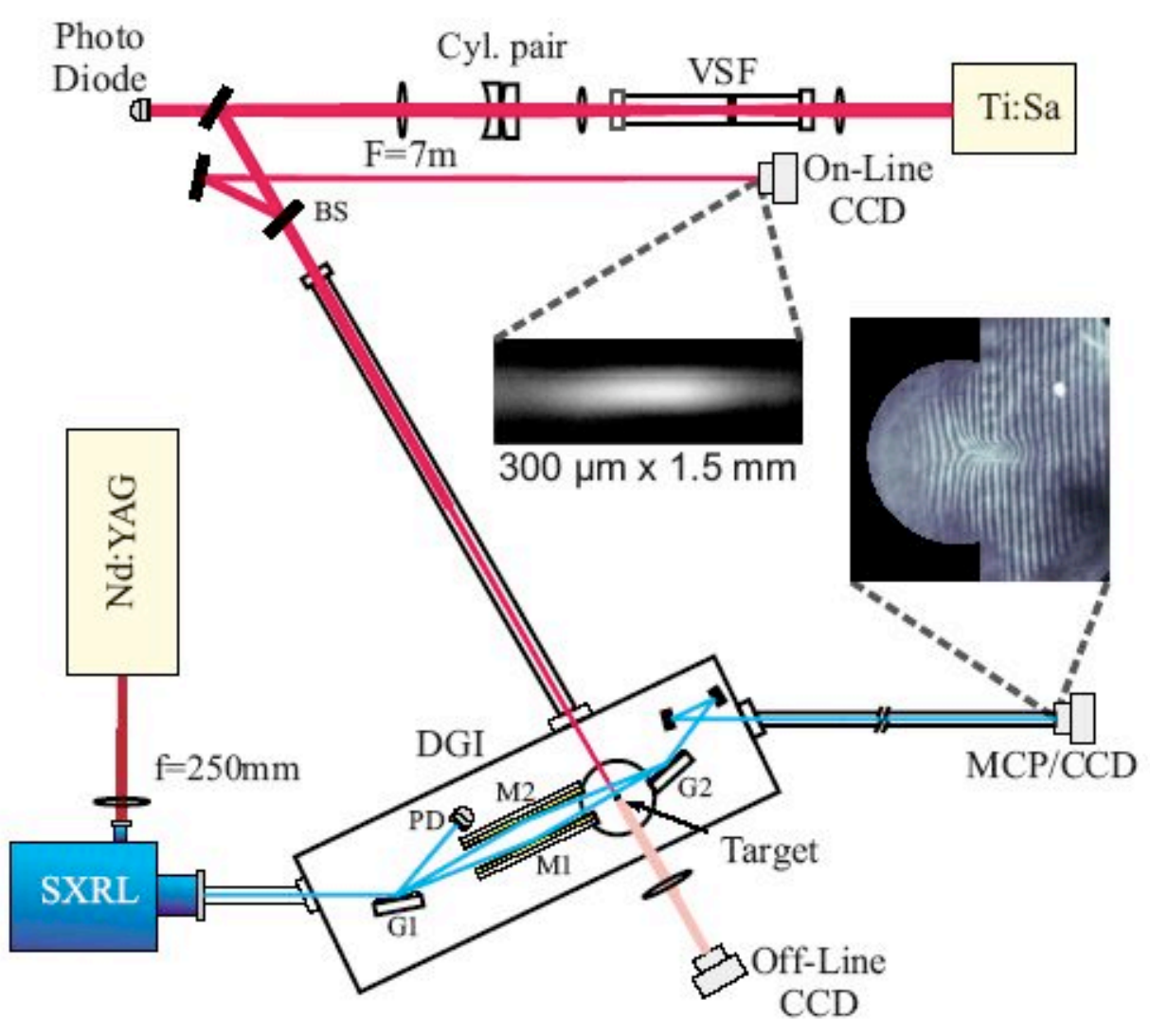

M. Purvis, J. Grava, J. Filevich, M. C. Marconi, J. J. Rocca, J. Dunn, S. J. Moon, V. N.

Shyaptsev, E. Jankowska", Phys. Rev. E 트, 046402 (2007) 
Carbon plasma converging plasma forms early $\sim 1 \mathrm{~ns}$ and is still visible at late times $\sim 17 \mathrm{~ns}$
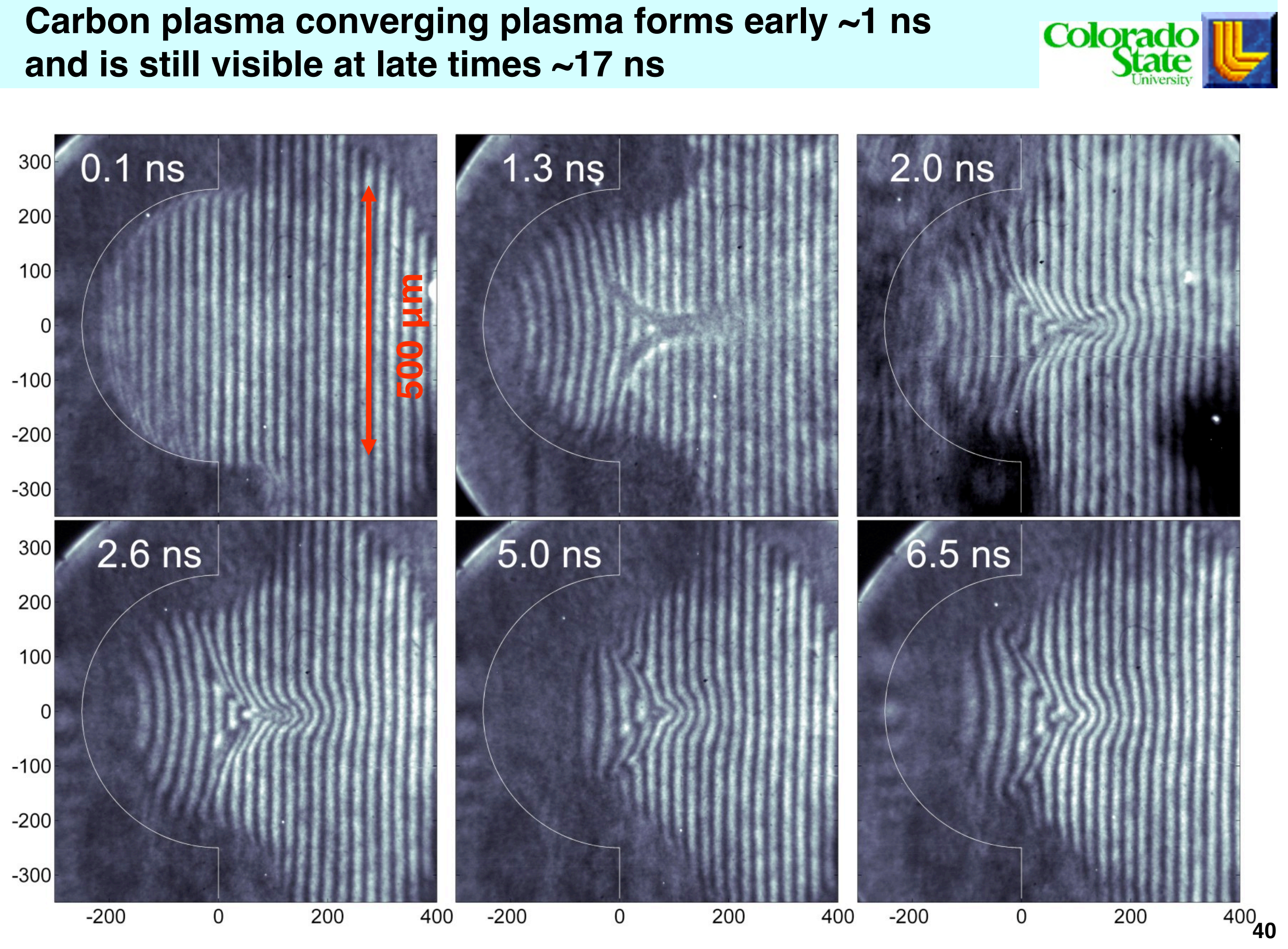
Comparison with HYDRA show excellent agreement in area where free electron contribution to fringes dominates

\section{Electron density maps obtained with the SXR interferometry}

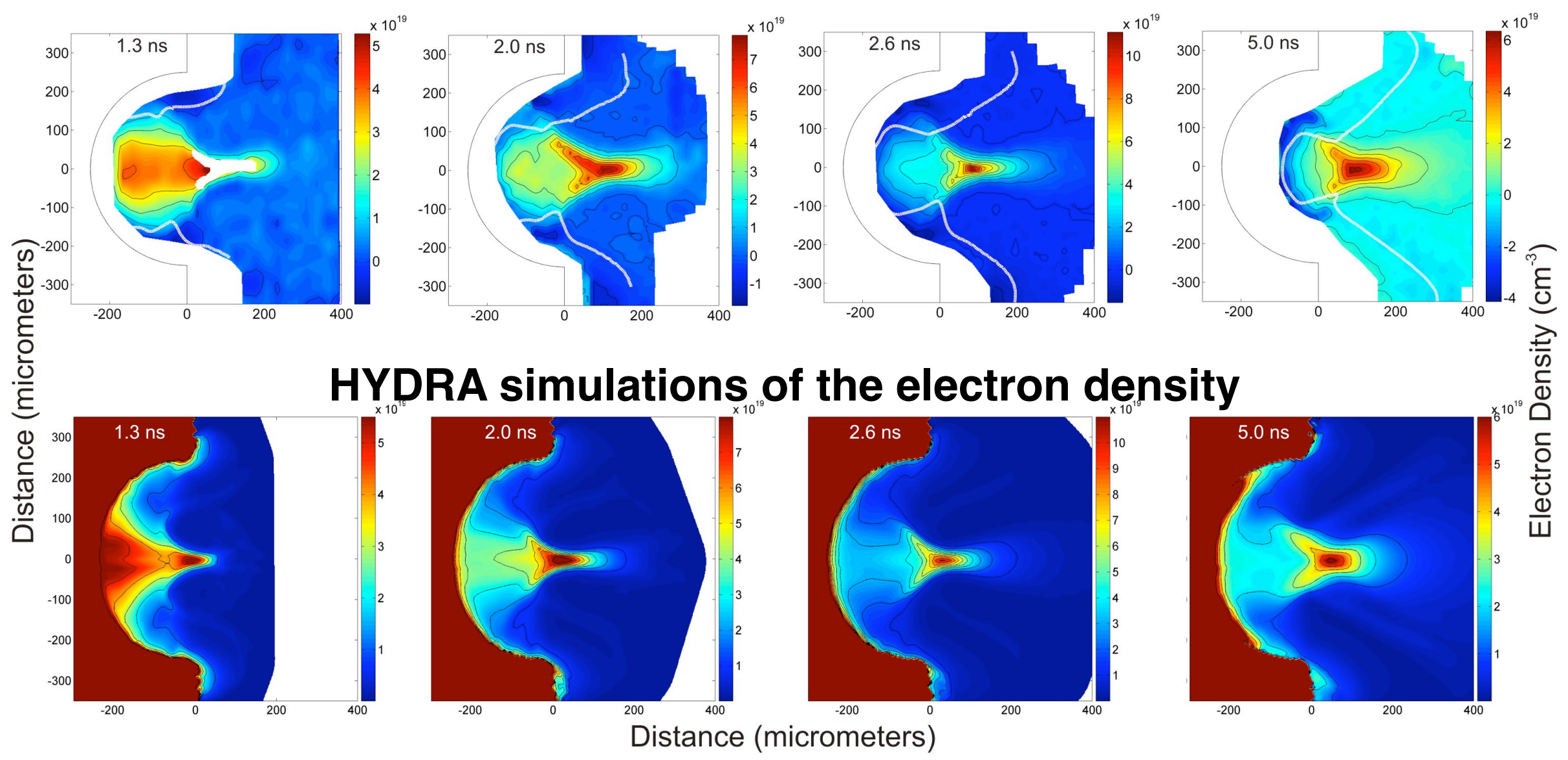

J. Filevich, J.J. Rocca, M.C. Marconi, S.J. Moon, J. Nilsen, J.H. Scofield, J. Dunn, R.F. Smith, R. Keenan, J.R. Hunter,V.N. Shlyaptsev, "Observation of a multiply ionized plasma with index of refraction greater than one", Phys. Rev. Lett. 94, 035005, (2005). 


\section{$\underline{\text { X-ray Laser Applications }}$}

- X-ray imaging and microscopy

- Material studies, ablation, nano-patterning 

been used in numerous applications

1. Interferometry of lasercreated plasmas

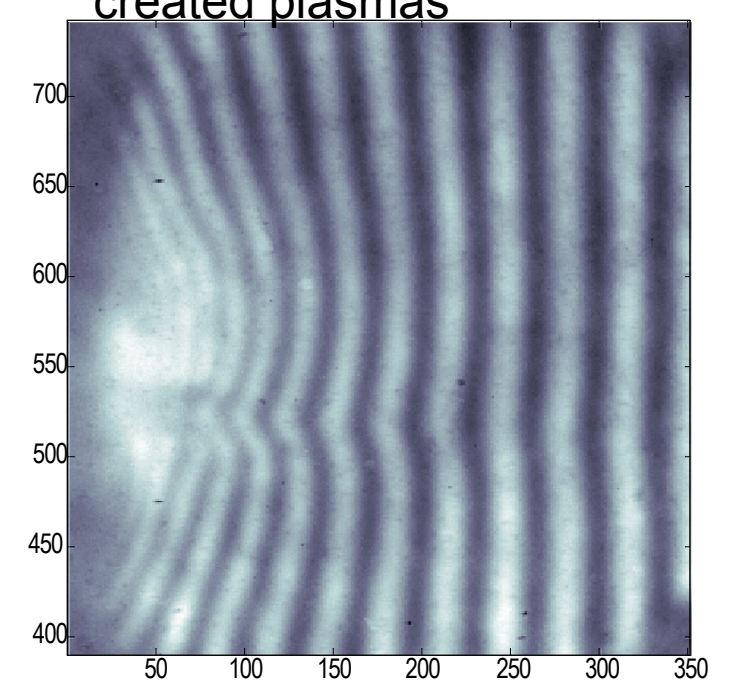

4. Laser Ablation

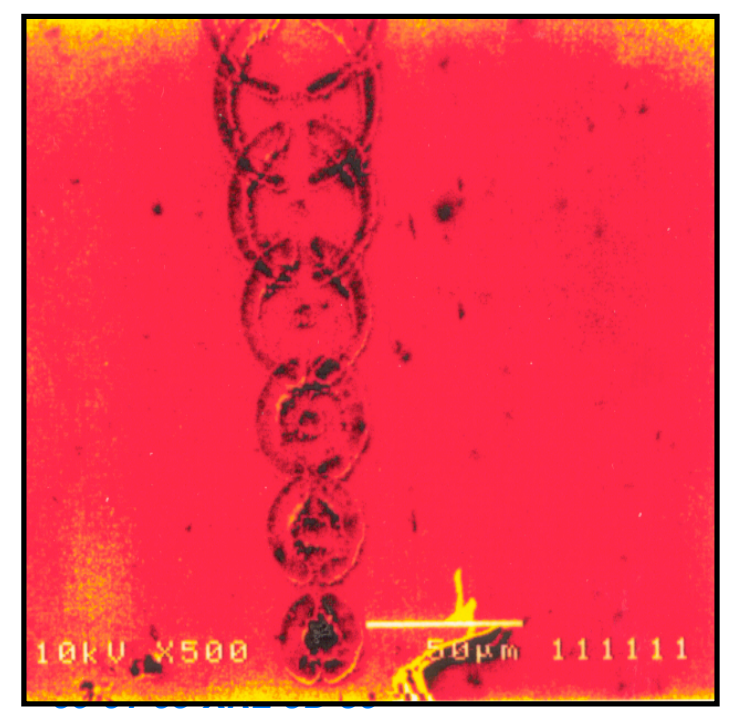

2. EUV microscopy

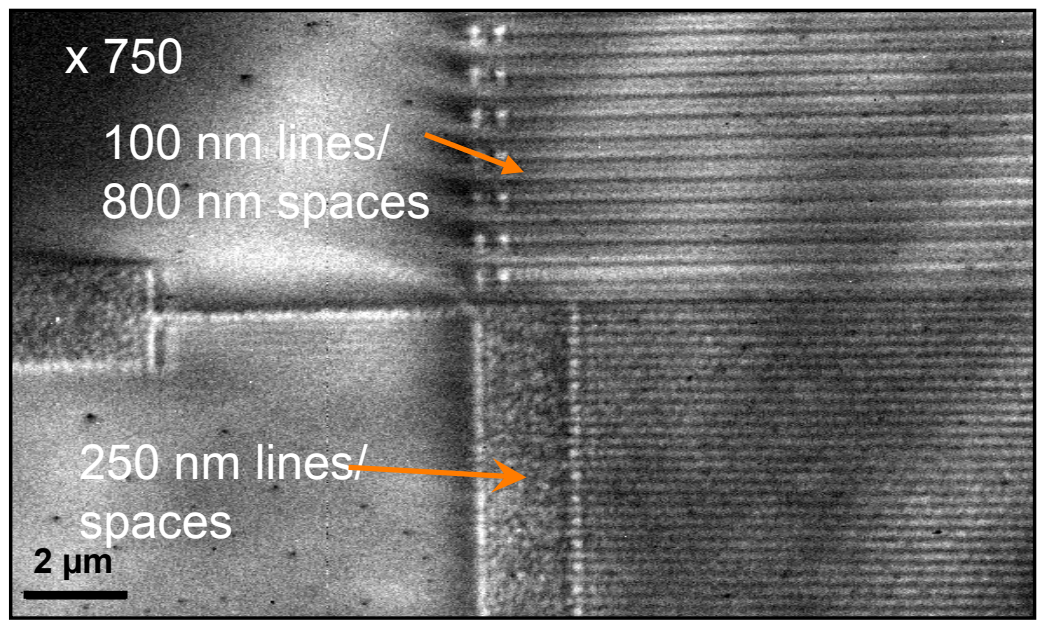

5. Nanocluster Spectroscopy

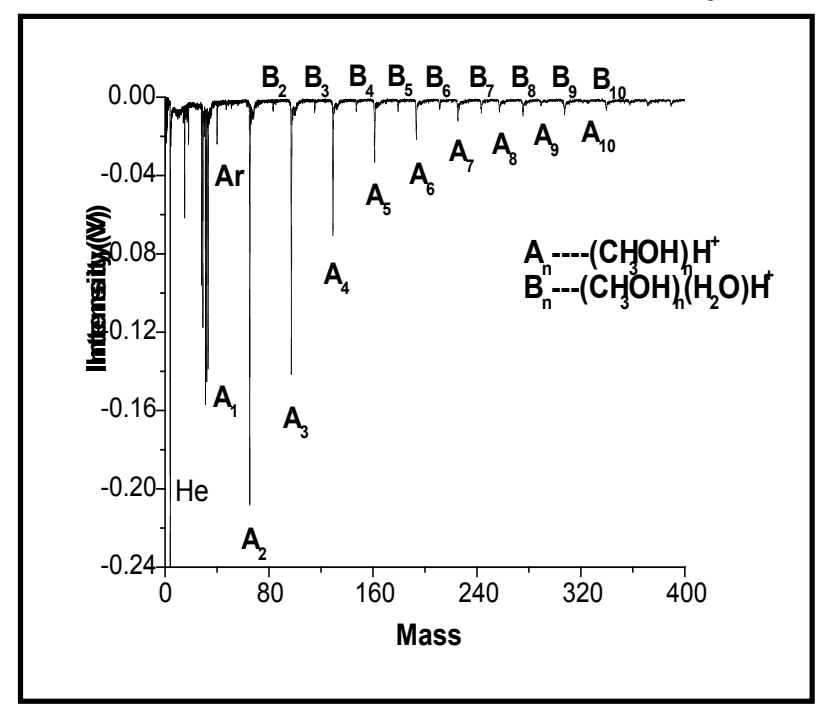

3. Nanopatterning

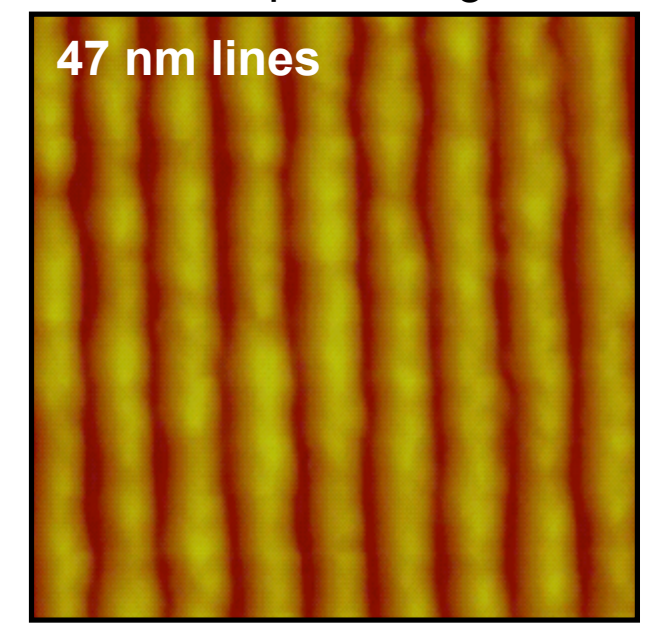

1. J.J. Rocca et al, Phys. Of Plasmas, 10, 2031 (2003).

2. F. Brizuela et al, Optics Express, 13, 3983, (2005)

3. M.G. Capeluto et al, IEEE Transactions on Nanotechnology, (in press),

4. J. Juha et al, Appl. Phys. Lett. 86, 034109 (2005).- M. Grisham et al, Optics Letters, $\underline{29}, 620$ (2004).
J. Rocca (CSU) 


\section{$\lambda=46.9 \mathrm{~nm}$ full field microscope operates in transmission and reflection configurations}

Colorado

\section{TRANSMISSION CONFIGURATION}

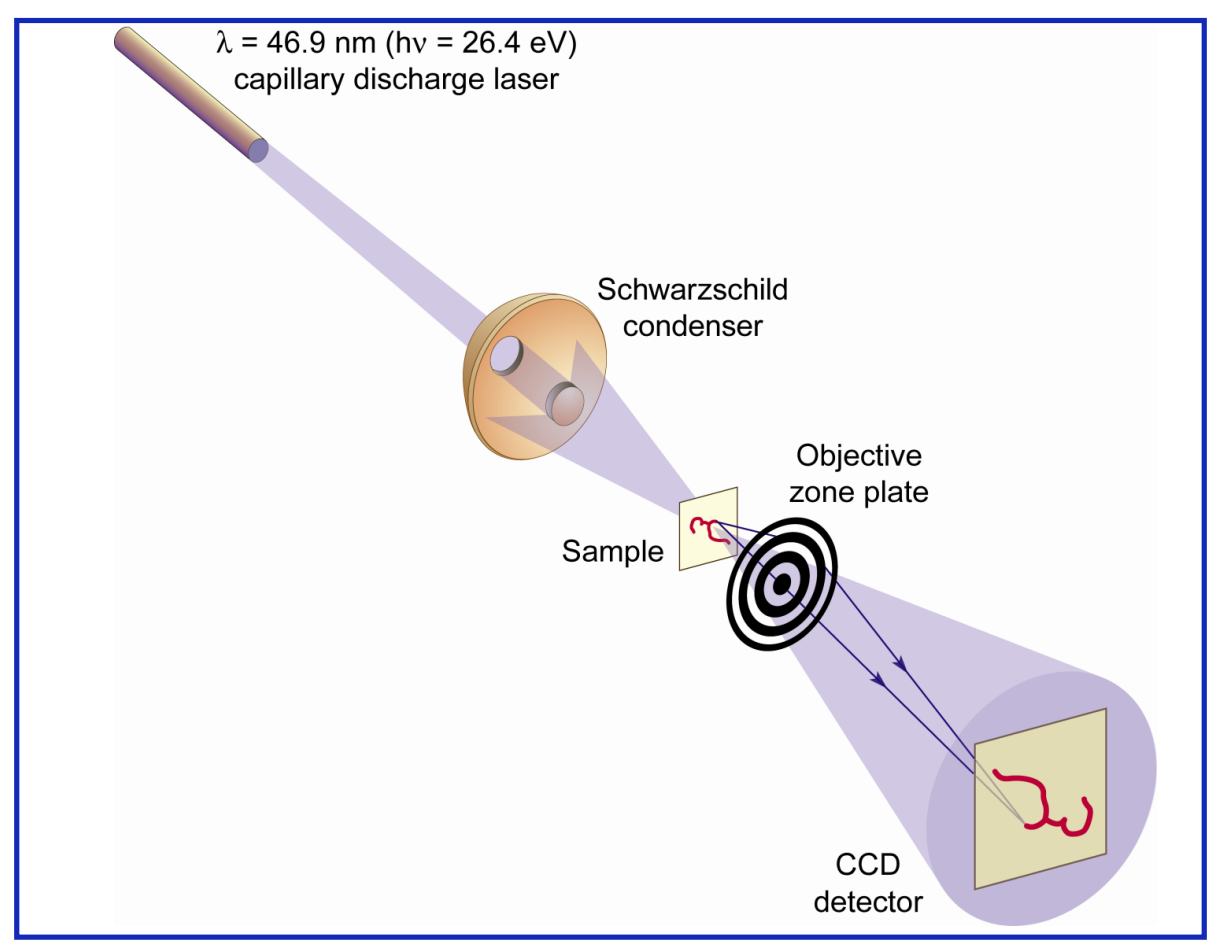

Spatial resolution: $50 \mathrm{~nm}$

Image capture time: single laser shot

\section{REFLECTION CONFIGURATION}

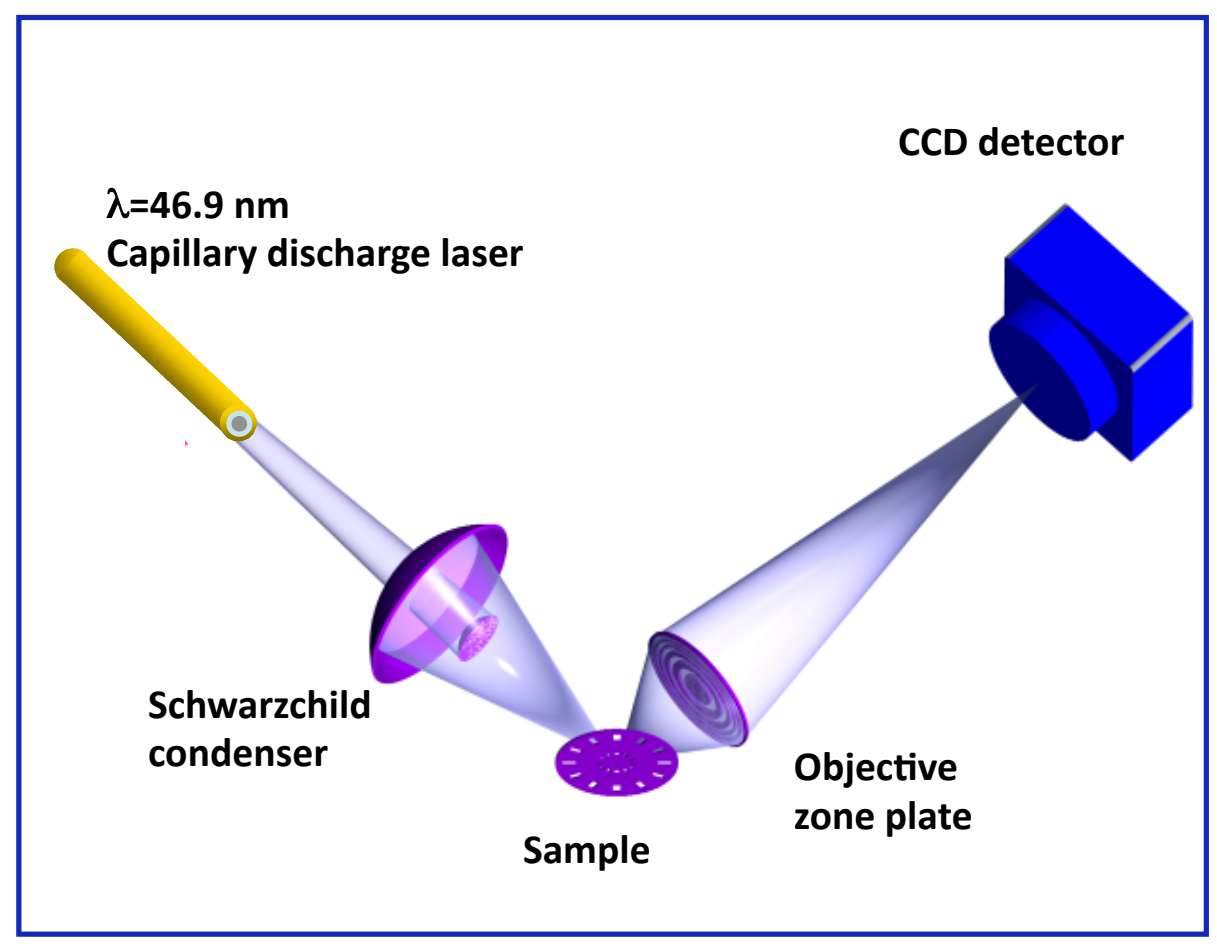

Spatial resolution: $80 \mathrm{~nm}$

Image capture time: 5-20 sec

C. Menoni, J. Rocca (CSU), D. Attwood (LBL) 
$46.9 \mathrm{~nm}$ microscope captures images of nanostructures with near-wavelength resolution

\section{TRANSMISSION \\ NA $=0.32(M \sim 1000)$}

$200 \mathrm{~nm}$ half period diatom

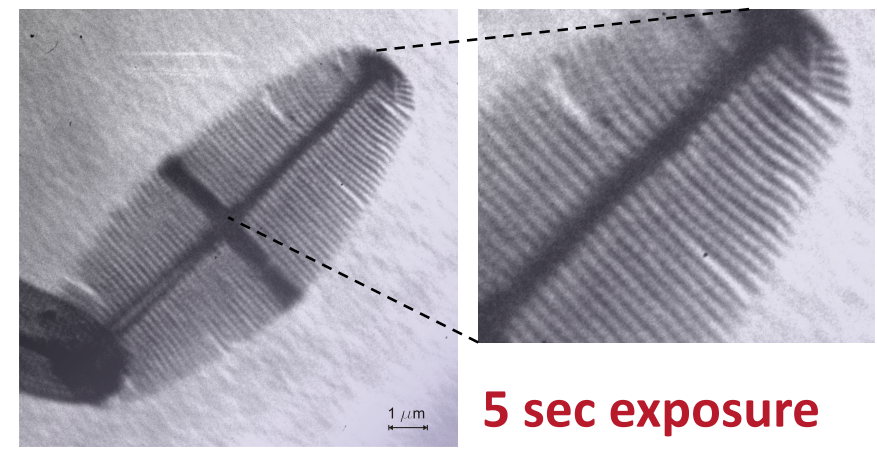

$50 \mathrm{~nm}$ carbon nanotube

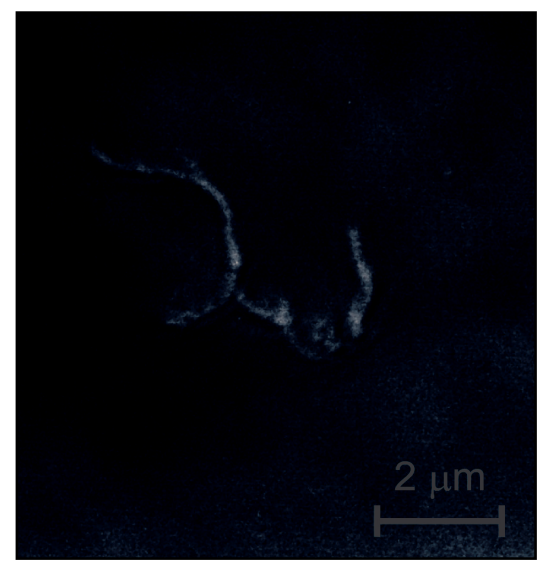

Single shot, exposure

$54 \mathrm{~nm}$ resolution

C. Brewer, et al, Opt. Lett. 33, 518 (2008)

\section{REFLECTION \\ $\mathrm{NA}=0.12$ and $0.19(\mathrm{M} \sim 250)$ \\ 5-20 sec exposures}

Partially Processed semiconductor chip

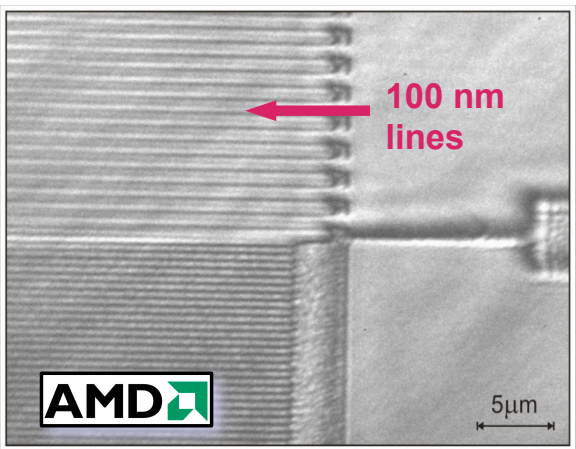

$100 \mathrm{~nm}$ thick GaN nanowire between Al contacts

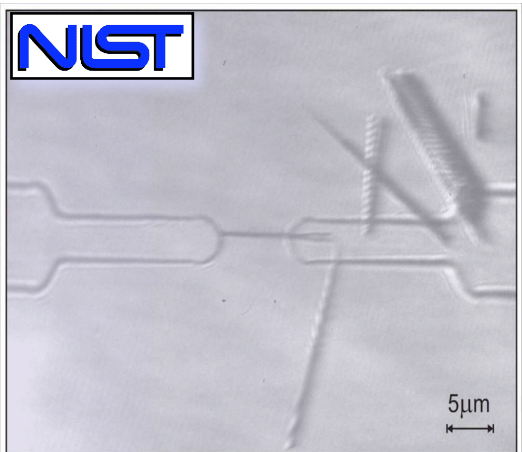

Zirconium surface

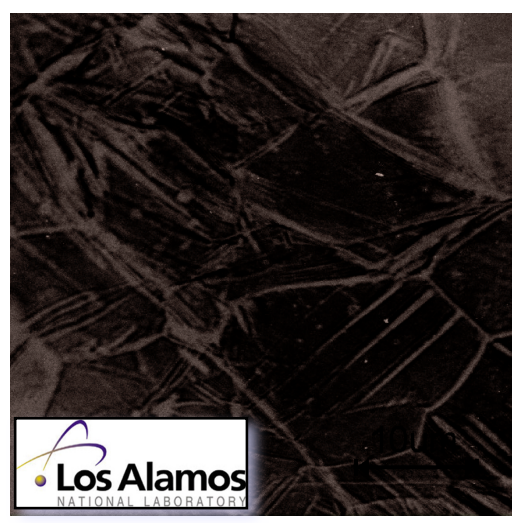

C. Menoni, J. Rocca (CSU), D. Attwood (LBL) 

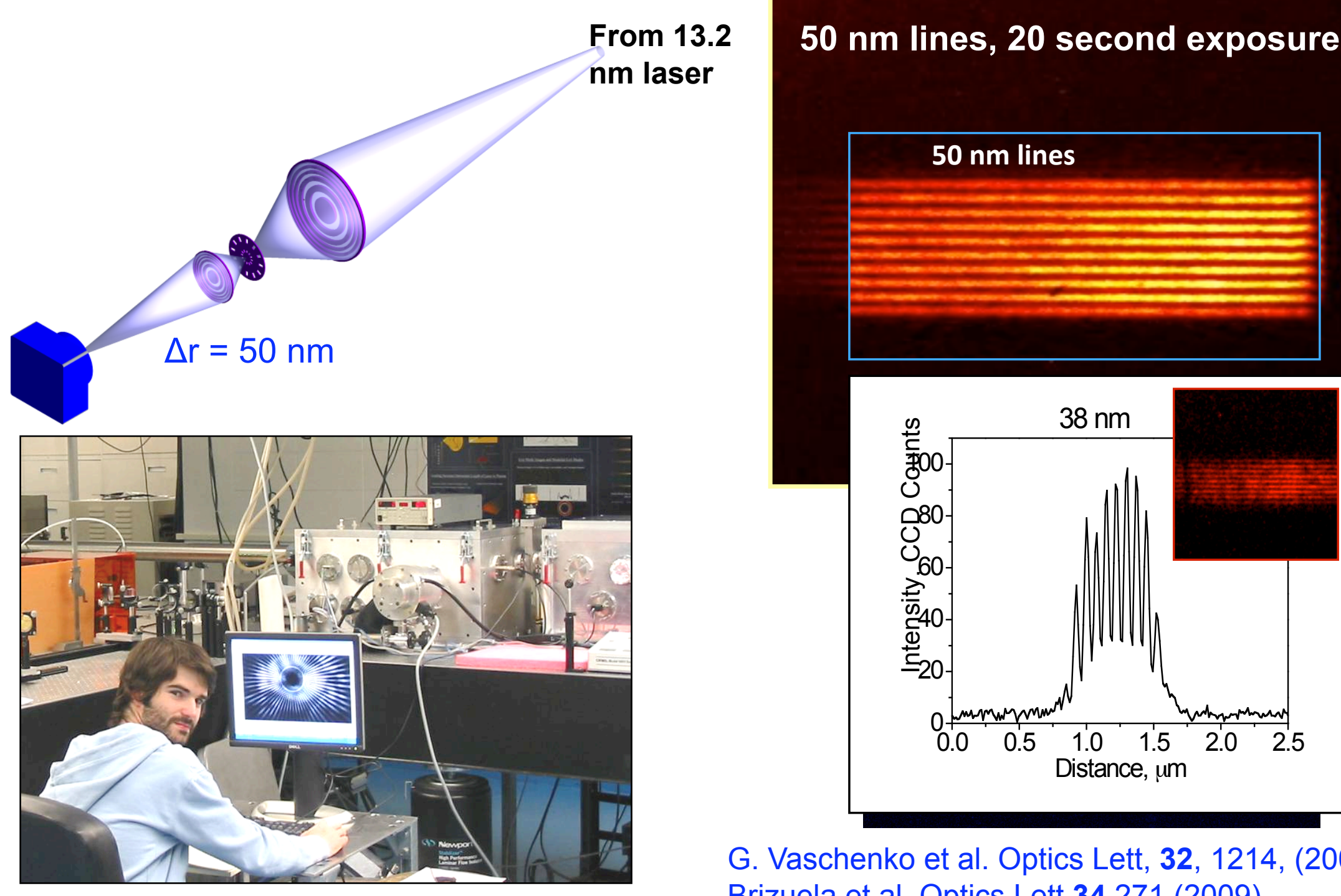

G. Vaschenko et al. Optics Lett, 32, 1214, (2006), F. Brizuela et al, Optics Lett,34,271 (2009)

C. Menoni, J. Rocca (CSU), D. Attwood (LBL) 

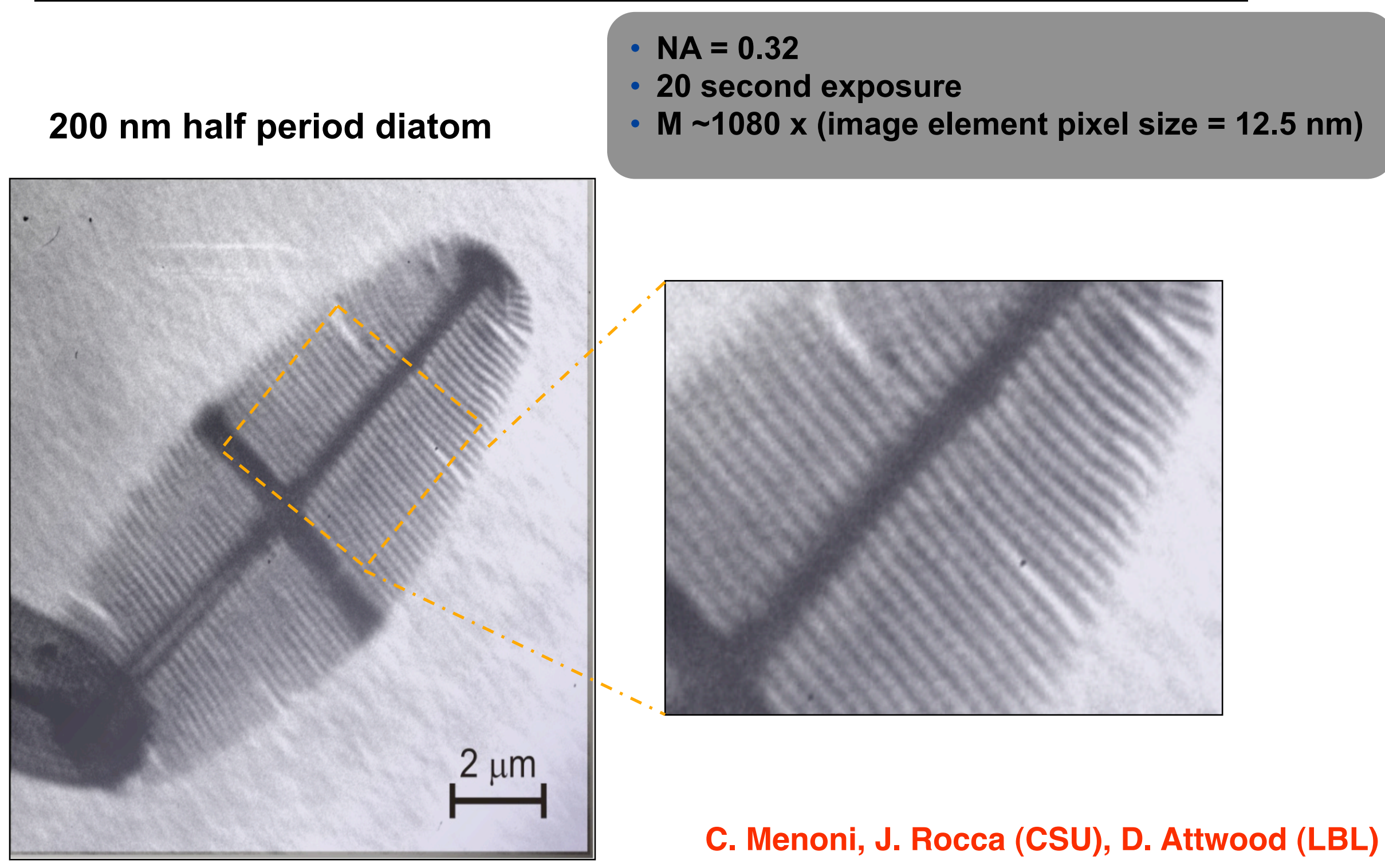

C. Menoni, J. Rocca (CSU), D. Attwood (LBL) 


\section{Lloyd's mirror interferometer} Step 1

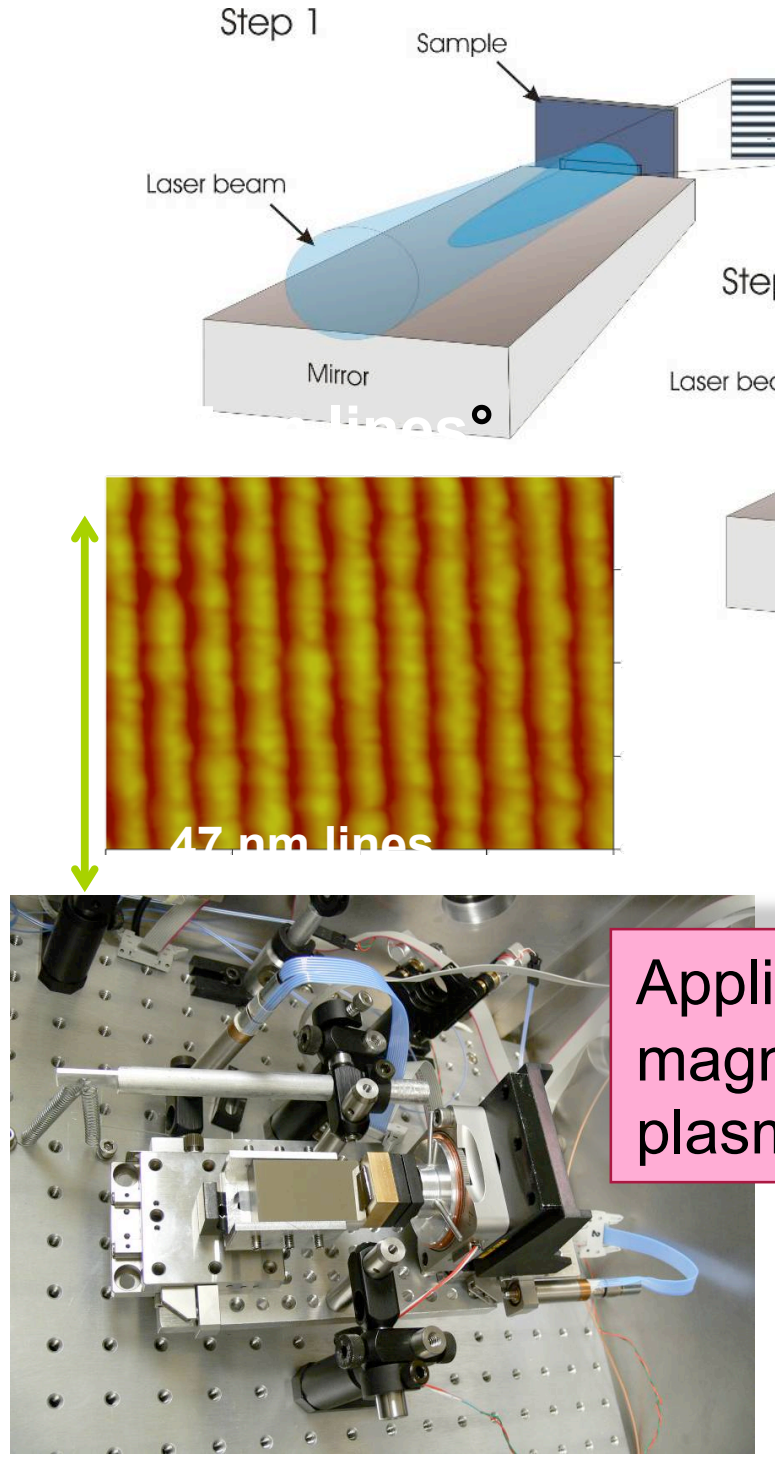

Array of $58 \mathrm{~nm}$ cones

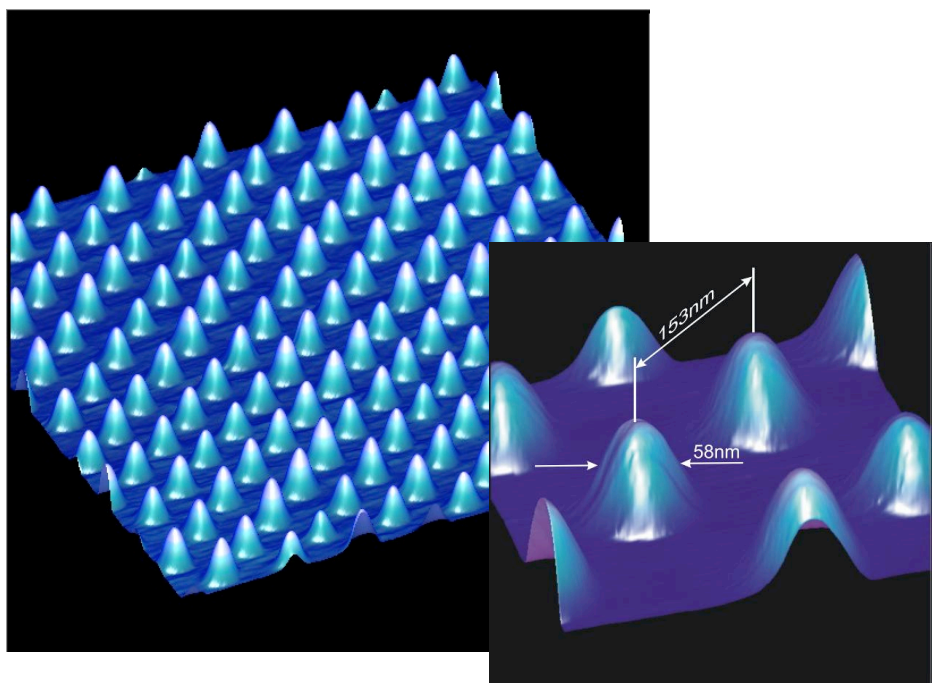

Array of $130 \mathrm{~nm}$ holes

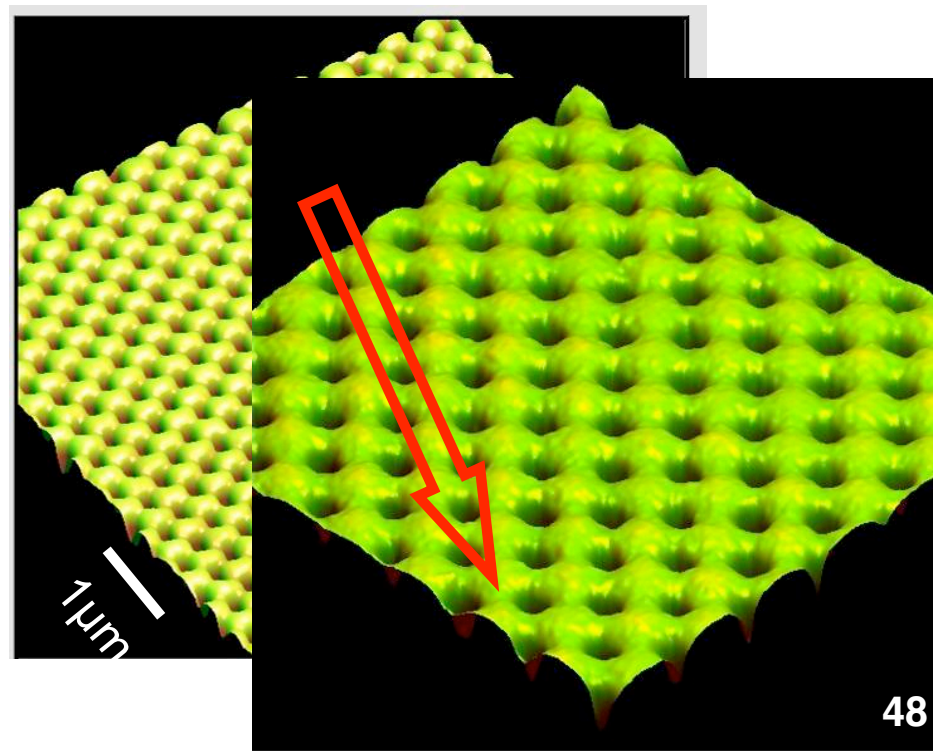




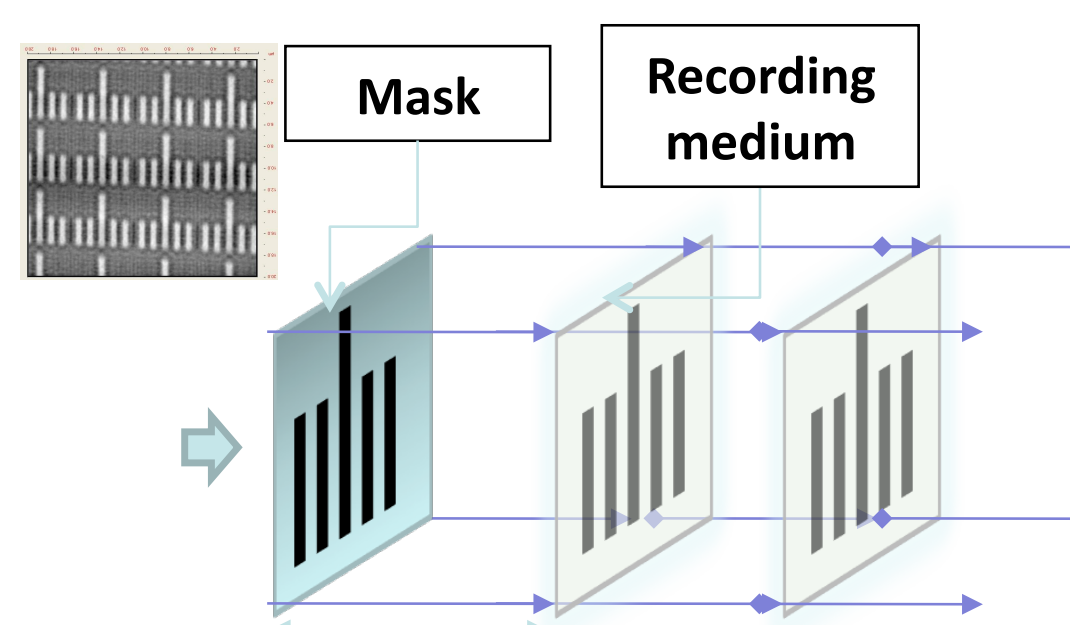

Talbot distance $D=\frac{2 \cdot(\text { Period })^{2}}{\lambda}$

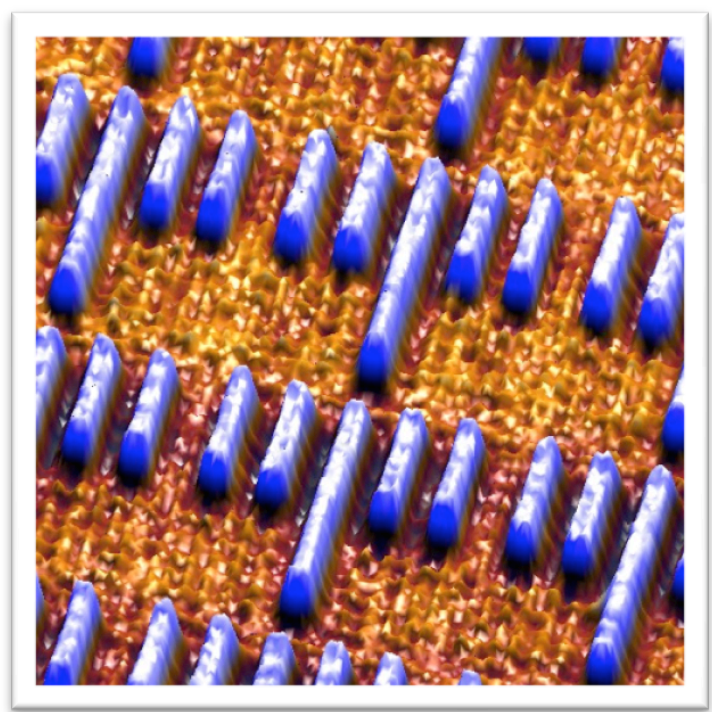

M. Marconi, F. Cerrina et al. in press JVST B

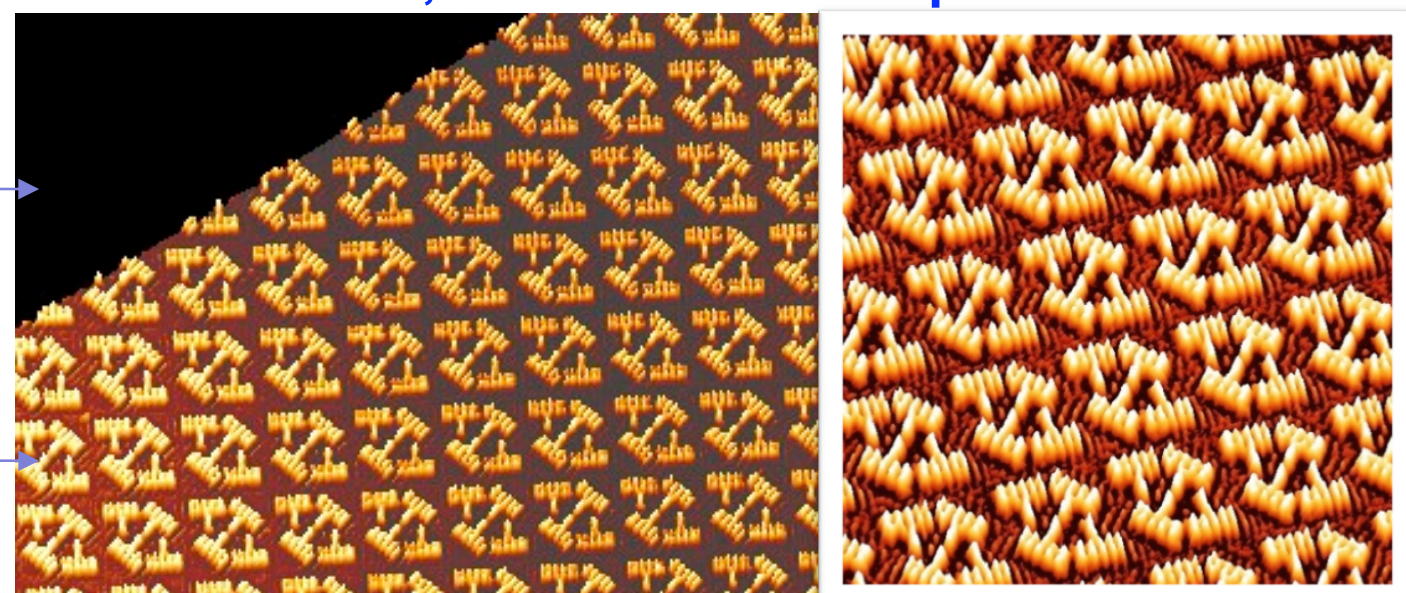

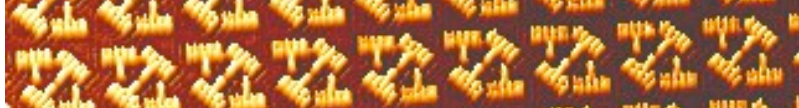

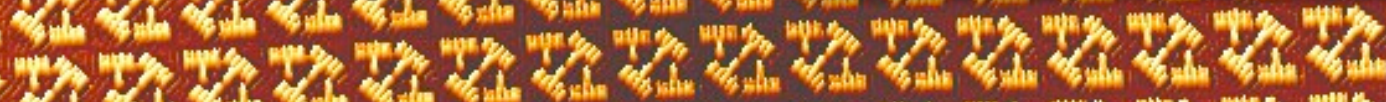
1.

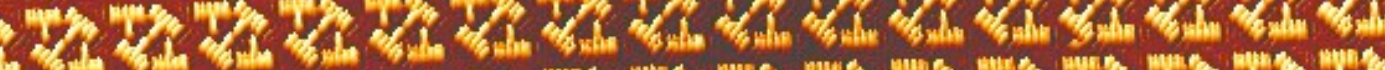

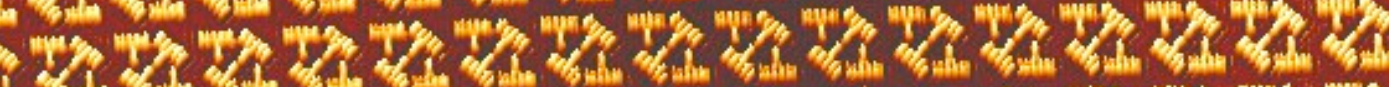

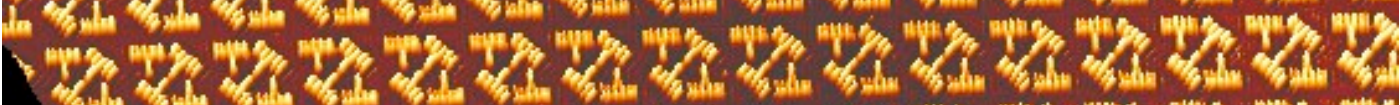

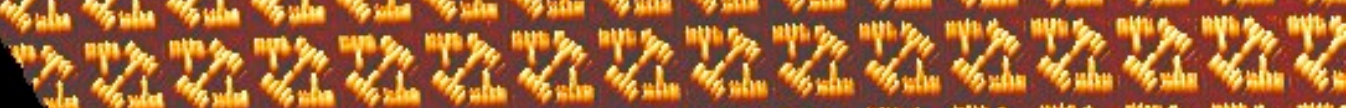

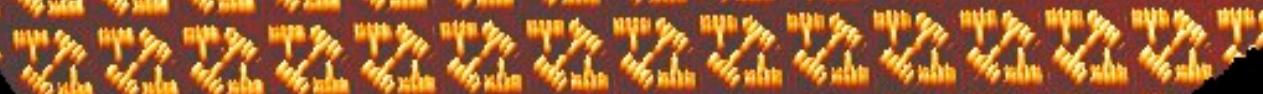

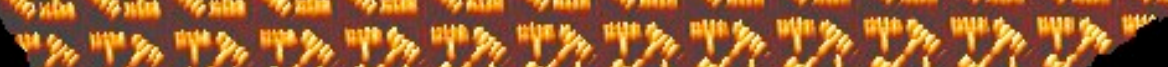

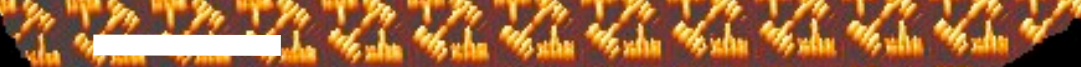

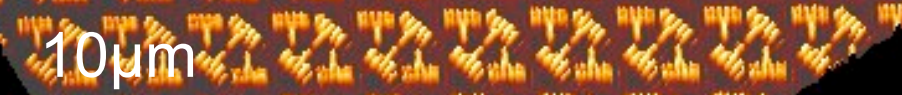

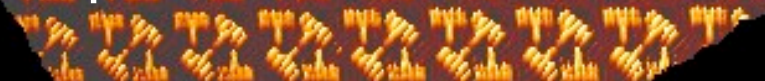




\section{Summary of Present Soft X-ray (EUV) Laser Characteristics:}

- Collisional excitation soft $x$-ray lasers:

$\lambda \sim 3.5 \mathrm{~nm}-60 \mathrm{~nm}(\mathrm{E} \sim 400 \mathrm{eV}-20 \mathrm{eV})$

- Characteristics:

- Gain saturation

Fs- Ps-Driven X-ray Lasers

PALS

$10 \mu \mathrm{J}$ output, $10^{12}$ photons/pulse

$10^{15} \mathrm{ph}$.

- High gain $30-80 \mathrm{~cm}^{-1}$ (small signal gain)

- Repetition rate

1 - $10 \mathrm{~Hz}$ (100 Hz DPSSL proposed by MBI, Berlin, CSU)

- Average Power

- Peak Power $\sim 20 \mu \mathrm{W} @ 13.9,13.2 \mathrm{~nm} @ 10 \mathrm{~Hz}, 1 \mathrm{~mW} @ 46.9 \mathrm{~nm}$

- Beam divergence

1 - $10 \mathrm{MW}$

$50-100 \mathrm{MW}$

$\sim 0.5$ - 2 mrad (FWHM)

- X-ray laser duration

1 - 8 ps (FWHM)

$100 \mathrm{ps}$

- Longitudinal coherence $\sim 400 \mu \mathrm{m}$ (1/e width)

- Spatial coherence $\quad \sim 3 \%$ for ASE amp. - full coherence for seeded

- Wave front

- Line width

$\lambda 17 @ 32.5$ nm

$\lambda \Delta \lambda=50,000$

- Brightness

$10^{25}-10^{26}$ ph. mm-2 $\mathrm{mrad}^{-2} \mathrm{~s}^{-1}[0.1 \% B W]^{-1}$

$10^{27}$

- Close to transform limited $\Delta E . \Delta t$ operation demonstrated 
Goal has been more efficient XRLs with lower drive energy and higher repetition rate: sub- $20 \mathrm{~nm}$ required

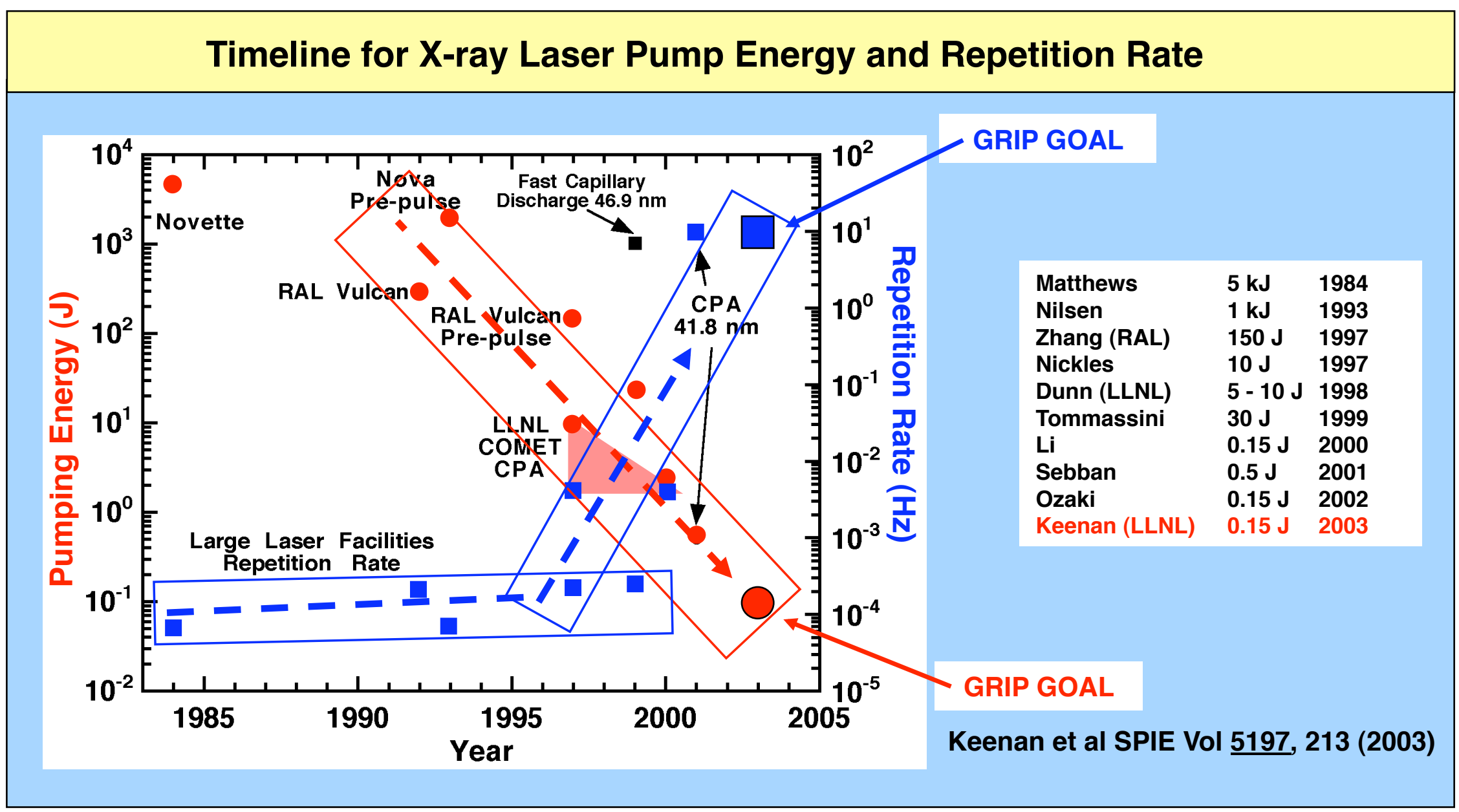

Achieved lasing with $\sim 10^{5}$ less energy compared to Nova

Many scientific applications require high repetition $\mathrm{x}$-ray sources 


\section{Conclusions and Future Outlook}

- Description and summary of plasma-based soft x-ray lasers (laser pumped)

- Recent developments in source generation: Smaller pump energy, higher repetition rate, improved beam qualities

- Examples of applications: Interferometry, ablation studies, probing matter

- Seeded Tabletop soft x-ray Lasers are direction of future

- Soft x-ray lasers are ultra-bright $10^{24}-10^{27} \mathrm{ph} . \mathrm{mm}^{-2} \mathrm{mrad}^{-2} \mathrm{~s}^{-1}[0.1 \% \mathrm{BW}]^{-1}$

- Latest generation are very compact (table top), inexpensive compared to synchrotrons, and are compatible with small research groups and universities

- Complement 3rd and 4th generation synchrotron sources for wavelength range, applications and regimes of interest:

- 100x narrower bandwidth than FELs coming online

- Full wavefront (spatial) coherence

- Potential for significantly higher output and brightness (stages) 